\title{
$\Gamma$-convergence of the Allen-Cahn energy with an oscillating forcing term
}

\author{
N. DIRR ${ }^{\dagger}$ \\ Max-Planck Institute for Mathematics in the Sciences, Inselstr. 22, D-04103 Leipzig, Germany \\ M. LUCIA ${ }^{\dagger}$ \\ Department of Mathematics, Hill Center, Rutgers University, \\ Piscataway, NJ 08854, USA \\ AND \\ M. NovAGA ${ }^{\S}$ \\ Dipartimento di Matematica, Università di Pisa, \\ Largo B. Pontecorvo 5, 56127 Pisa, Italy
}

[Received 5 April 2005 and in revised form 21 September 2005]

\begin{abstract}
We consider a standard functional in the mesoscopic theory of phase transitions, consisting of a gradient term with a double-well potential, and we add to it a bulk term modeling the interaction with a periodic mean zero external field. This field is amplified and dilated with a power of the transition layer thickness $\epsilon$ leading to a nontrivial interaction of forcing and concentration when $\epsilon \rightarrow 0$. We show that the functionals $\Gamma$-converge after additive renormalization to an anisotropic surface energy, if the period of the oscillation is larger than the interface thickness. Difficulties arise from the fact that the functionals have nonconstant absolute minimizers and are not uniformly bounded from below.
\end{abstract}

\section{Introduction}

We briefly review some aspects of the classical theory of phase transitions. Given $\Omega \subset \mathbb{R}^{N}$, let $u: \Omega \rightarrow \mathbb{R}$ be an order parameter, i.e. a function which describes to what extent the physical system at a given point $x \in \Omega$ is in the "+" or "-" phase. Pure phases correspond to the two minimizers (for instance \pm 1 ) of a double-well potential $W$, which can be derived from atomistic considerations as a mean-field free energy, and whose main property is to be convex in a neighborhood of \pm 1 . The resulting free energy functional is characterized by a competition between a gradient term, modeling interaction energy, and the potential $W$. Such a functional is given by

$$
M_{\epsilon}(u):=\int_{\Omega}\left\{\epsilon|\nabla u|^{2}+\frac{W(u)}{\epsilon}\right\} \mathrm{d} x, \quad u \in H^{1}(\Omega),
$$

where $\epsilon>0$ is a small parameter related to the interface thickness. If the system is prevented from staying close to +1 or to -1 everywhere (for example by a volume constraint), then the thickness

\footnotetext{
${ }^{\dagger}$ Supported by the German Academic Exchange Service (DAAD) and the Department of Mathematics of the University of Texas at Austin. E-mail: ndirr@mis.mpg.de

E-mail: mlucia@math.rutgers.edu

${ }^{\S}$ E-mail: novaga@mail.dm.unipi.it
} 
of the transition layer (i.e. the set separating the positive and negative regions) will be of order $\epsilon$. Moreover, sequences of finite energy for $\epsilon \rightarrow 0$ should converge to \pm 1 almost everywhere.

A suitable mathematical setup to make this rigorous is the notion of $\Gamma$-convergence (see Section 2 for a precise definition). In [16, 15] the authors characterize the $\Gamma$-convergence of the family $M_{\epsilon}$ with respect to the $L^{1}(\Omega)$-topology and they obtain a sharp interface limit, which is the area of the interface with surface tension $c_{W}$ (which is related to the double-well potential). More precisely, by setting

$$
c_{W}:=\int_{-1}^{1} \sqrt{W(t)} \mathrm{d} t \quad \text { and } \quad \mathcal{B}:=\{u \in B V(\Omega): u(x) \in\{-1,1\} \text { a.e. in } \Omega\},
$$

they prove that the $\Gamma$-limit of the functionals in 1.1 , extended by $+\infty$ to all $L^{1}(\Omega)$, is given by

$$
M_{0}(u):= \begin{cases}c_{W} P(E, \Omega) & \text { if } u=\chi_{E} \in \mathcal{B}, \\ +\infty & \text { if } u \in L^{1}(\Omega) \backslash \mathcal{B} .\end{cases}
$$

This convergence could be perturbed by rapidly oscillating spatial inhomogeneities modeling for example the interaction with a substrate. The result will depend on whether the scale on which the inhomogeneities oscillate is of order of the interface thickness, smaller or larger. One way to introduce spatial inhomogeneities is to consider an $x$-dependent gradient term, i.e. replace the term $|\nabla u|^{2}$ in 1.1 by $\left|A\left(x / \epsilon^{\alpha}\right) \nabla u\right|^{2}$, where $A(x)$ is a positive definite symmetric matrix, periodically depending on $x$ (a general version of this case is studied in [2]). In our paper, instead, the energy in (1.1) is perturbed by a strong, rapidly oscillating field with zero average. More precisely, we shall consider the functional

$$
G_{\epsilon}(u):=\int_{\Omega}\left\{\epsilon|\nabla u(x)|^{2}+\frac{W(u(x))}{\epsilon}+\frac{1}{\epsilon^{\alpha}} g\left(\frac{x}{\epsilon^{\alpha}}\right) u(x)\right\} \mathrm{d} x, \quad u \in H^{1}(\Omega),
$$

where $g \in L^{\infty}\left(\mathbb{R}^{N}\right)$ is a periodic function with cell domain $Q:=(-1 / 2,1 / 2)^{N}$. This periodic term $g$ has the effect of creating many local minima. Systems of this type are of relevance in materials science, e.g. the evolution of microstructures or the motion of magnetic walls.

When $\alpha=0$, it follows from the results in [16, 15] (see also [9, Proposition 6.21]) that the $\Gamma$-limit is the sum of the functional 1 1.2) and the volume term $\int g(x) u(x) \mathrm{d} x$. When $\alpha>0$, both amplitude and frequency of $g$ become large as $\epsilon \rightarrow 0$, hence the infimum of the functional over $H^{1}(\Omega)$ can be negative or even converge to $-\infty$ as $\epsilon \rightarrow 0$ (for example when $\alpha>1 / 2$, see Proposition 3.9]. Therefore, to fit in the framework of $\Gamma$-convergence, we need to introduce an additive renormalization. However, in order to get a nontrivial $\Gamma$-limit, we need the renormalization to be of the same order of the perimeter and this can happen only if $\int_{Q} g \mathrm{~d} x=0$. We show for $0<\alpha<1$ that the renormalized functionals $\Gamma$-converge to an anisotropic surface energy (see Theorem 2.3.

There are similarities with the result in [2] but in many respects our setting requires new techniques. The main difficulties (beyond those encountered in [16, 15] and [2]) arise from this renormalization and the (related) facts that the functionals have nonconstant global minimizers whose energy is not uniformly bounded from below. To explain the main points, let us first note that the Euler-Lagrange equation is

$$
\epsilon \Delta u-\frac{W^{\prime}(u)}{2 \epsilon}=\frac{1}{2 \epsilon^{\alpha}} g\left(\frac{x}{\epsilon^{\alpha}}\right) \quad \text { on } \Omega, \quad \frac{\partial u}{\partial n}=0 \quad \text { on } \partial \Omega,
$$


i.e. the function $g$ appears as a forcing term. There are two solutions of (1.3): $u_{\epsilon}^{+}$, close to +1 , and $u_{\epsilon}^{-}$, close to -1 (see Proposition 3.7 and Corollary 3.8), which are local minimizers of the energy and which are nonconstant if $g \not \equiv 0$, whereas in the unperturbed case or in [2] one gets $u_{\epsilon}^{+} \equiv 1, u_{\epsilon}^{-} \equiv-1$. As their energy is strictly negative, and typically is of order $|\Omega| \epsilon^{1-2 \alpha}$, the aforementioned additive renormalization is necessary.

The appearance of such a renormalization is in fact quite natural for phase transition problems. The energy associated with an interface is the excess free energy due to the fact that more than one phase is present, so it is actually a difference of energies, determined only up to adding constants. If the pure phases, i.e. the global minimizers, are constants, then in order to ensure that the energy of the minimizers is zero, it is enough to choose $\min _{\mathbb{R}} W(u)=0$. In our case the minimizers are not constants, so we must compute their energy and show that it is proportional to the volume of the domain $\Omega$ (up to smaller order), as we want a local functional as $\Gamma$-limit. Moreover (again up to smaller order) the energy of $u^{+}$and $u^{-}$must be the same. Conditions on $W$ and $g$ will ensure both these properties.

Now we consider the different scalings, i.e. the oscillation of $g$ in relation to the interface thickness $\epsilon$. In this paper we treat rigorously the case of slow oscillations, i.e. $0<\alpha<1$, leaving the case $\alpha \geqslant 1$ to further investigation. Let $\gamma: \mathbb{R} \rightarrow[-1,1]$ be the unique increasing solution of

$$
2 \gamma^{\prime \prime}=W^{\prime}(\gamma)
$$

which converges exponentially to \pm 1 at $\pm \infty$, and such that $\gamma(0)=0$. If we perform the change of variables $y=x \epsilon^{-\alpha}$ and let $\tilde{u}(y)=u\left(x \epsilon^{-\alpha}\right),(1.3)$ becomes

$$
\epsilon^{1-\alpha} \Delta \tilde{u}-\frac{W^{\prime}(\tilde{u})}{2 \epsilon^{1-\alpha}}=\frac{1}{2} g(y) .
$$

Then a formal asymptotic expansion for solutions of 1.5 gives

$$
\tilde{u}(y)=\gamma\left(\frac{\tilde{d}(y)}{\epsilon^{1-\alpha}}\right)+\epsilon^{1-\alpha} \tilde{u}_{1}\left(\sigma(y), \frac{\tilde{d}(y)}{\epsilon^{1-\alpha}}, y\right)+o\left(\epsilon^{1-\alpha}\right),
$$

where $\tilde{d}(x)$ is the signed distance function from the zero-level set of $\tilde{u}$ (which we assume to be a smooth hypersurface) and $\sigma(y):=y-\tilde{d}(y) \nabla \tilde{d}(y)$ is the projection of $y$ onto $\{\tilde{u}=0\}$. It follows that $c_{W} \Delta \tilde{d}(x)=g(x)$ on $\{\tilde{u}=0\}$, which on the original scale becomes

$$
c_{W} \kappa=\frac{1}{\epsilon^{\alpha}} g\left(\frac{x}{\epsilon^{\alpha}}\right)
$$

where $\kappa$ is the mean curvature of the zero-level set of $u$. Hence, for $\alpha<1$ the problem is related to singular homogenization for the prescribed mean curvature equation. Indeed, in this case there is a splitting of the $\Gamma$-limit into a more standard limit, similar to [16, 15] with a $g$-term which does not depend on $\epsilon$, and a prescribed mean curvature problem (see Theorems 2.3 and 5.9 ).

Equation (1.6) shows that the chosen relation between amplitude and frequency of the forcing term is interesting, since the interface will change its shape significantly within one unit cell. For a stronger amplitude we expect to see small bubbles everywhere, as the minimizers on a cell are no longer of constant sign, whereas for a weaker forcing the limit will be isotropic.

Now we are able to summarize our results. Any sequence of bounded energy has a subsequence which converges in $L^{1}$ to a BV-function, which takes its values in $\{-1,1\}$. The $\Gamma$-limit with respect 
to $L^{1}$-convergence has the form

$$
\int_{\partial^{*} E \cap \Omega} \varphi\left(v_{E}\right) \mathrm{d} \mathcal{H}^{N-1}
$$

where $E$ is a finite perimeter set on which $\lim u^{\epsilon}=1$ and $v$ is the unit normal to $\partial^{*} E$. Thanks to the aforementioned splitting, the anisotropy $\varphi$ can be explicitly characterized (see Theorem 5.9p, and $\varphi(\nu)=\varphi(-v)$ and $\varphi \leqslant c_{W}$ for any forcing term $g$ satisfying certain bounds and a symmetry condition (see Proposition 5.11).

Note that this is not a $\Gamma$-limit result for the functionals $G_{\epsilon}$ but only for the renormalized functionals, since the functionals $G_{\epsilon}$ typically converge to $-\infty$ when $\alpha>1 / 2$ (see the comments after Proposition 2.2. Such a result is more in the spirit of a $\Gamma$-expansion, as recently investigated in [7].

We add a few comments on the case of fast oscillations, i.e. $\alpha \geqslant 1$. When $\alpha=1$, there is no splitting of scales as before, hence this case is more difficult. However, under possibly stronger conditions on $g$, we expect a similar $\Gamma$-convergence result to hold, and that the limit is still an anisotropic surface energy. For $\alpha>1$, we expect the limit functional to be isotropic, i.e. a multiple of the usual perimeter.

The paper is organized as follows. In Section 2, we briefly review the theory of $\Gamma$-convergence, following [9]. Moreover, we state our assumptions on $W$ and $g$, we define the renormalized functionals and we give a precise statement of the main result. In Section 3, we show the existence of the minimizers $u_{\epsilon}^{ \pm}$and estimate the cost of having a transition within a cube. In Section 4 , we show that any sequence with bounded energy has a subsequence converging in $L^{1}(\Omega)$ to a $B V$ function taking values only in $\{-1,1\}$. Using the estimates of Section 3 , we derive the so-called "fundamental estimate", which is a localization property. We also show that the limit energy of our functionals concentrates on characteristic functions and is bounded from above and below by the area functional.

General principles allow us to derive from these estimates a first $\Gamma$-limit theorem, which is valid up to a subsequence (see Proposition 4.11). In Section 5, we derive further properties of the limit functional and obtain, in particular, a representation formula (see Theorem 5.9), which implies that the $\Gamma$-limit is independent of the subsequence and of the scale parameter $\alpha$.

\section{Notation and main results}

Let $N \geqslant 2$. We denote by $\mathcal{A}$ the class of all bounded open subsets of $\mathbb{R}^{N}$ and by $Q:=(-1 / 2,1 / 2)^{N}$ the open unit cube in $\mathbb{R}^{N}$ centered at 0 . For each $E \subset \mathbb{R}^{N}$, the (shifted) characteristic function $\chi_{E}$ of $E$ and the (signed) distance function $d_{E}$ from $\partial E$ are defined respectively by:

$$
\chi_{E}(x):=\left\{\begin{array}{ll}
1 & \text { if } x \in E, \\
-1 & \text { otherwise, }
\end{array} \quad d_{E}(x):= \begin{cases}-\operatorname{dist}\left(x, \mathbb{R}^{N} \backslash E\right) & \text { if } x \in E, \\
\operatorname{dist}(x, E) & \text { otherwise. }\end{cases}\right.
$$

Moreover, if $E \subseteq \Omega \in \mathcal{A}$ with $\chi_{E} \in B V(\Omega)$, we denote by $P(E, \Omega)$ the perimeter of $E$ in $\Omega$, and by $\partial^{*} E$ the reduced boundary of $E$ (see [12]). Given $u \in B V(\Omega)$, we denote by $\int_{\Omega}|\nabla u|$ the total variation of $u$ in $\Omega$, thus we have

$$
\int_{\Omega}\left|\nabla \chi_{E}\right|=P(E, \Omega)
$$

for all $E \subseteq \Omega$ of finite perimeter in $\Omega$. Let also briefly recall the notion of $\Gamma$-convergence (see [9] for more details on this subject). 
Definition 2.1 Let $X$ be a metric space and let $F_{\epsilon}: X \rightarrow \overline{\mathbb{R}}, \epsilon>0$, be a family of functionals on $X$. We say that $F_{\epsilon} \Gamma$-converge to $F: X \rightarrow \overline{\mathbb{R}}$ if the following conditions are satisfied:

1. for all $x \in X$ and for all $x_{\epsilon} \rightarrow x$,

$$
\liminf _{\epsilon \rightarrow 0} F_{\epsilon}\left(x_{\epsilon}\right) \geqslant F
$$

( $\Gamma$-liminf inequality);

2. for all $x \in X$ there exist $x_{\epsilon} \rightarrow x$ such that

$$
\lim _{\epsilon \rightarrow 0} F_{\epsilon}\left(x_{\epsilon}\right)=F
$$

( $\Gamma$-limsup inequality).

We recall the following fundamental property of $\Gamma$-convergence, which can be easily derived from Definition 2.1.

Proposition 2.2 If $F_{\epsilon} \Gamma$-converge to $F$ in $X$, also the corresponding minimal values (or infima) converge. Moreover, if $x_{\epsilon}$ is a minimizer of $F_{\epsilon}$ and $x_{\epsilon} \rightarrow x \in X$, then $x$ is a minimizer of $F$.

Hence, the asymptotic behavior of minimizers of $F_{\epsilon}$ can be partly understood by considering the $\Gamma$-limit of $F_{\epsilon}$. Notice also that the second assertion of Proposition 2.2 does not change if we modify the functionals $F_{\epsilon}$ by adding a constant (renormalization), possibly depending on $\epsilon$.

Given $\Omega \in \mathcal{A}$ and $\epsilon>0$, we consider the following functional:

$$
G_{\epsilon}(u, \Omega):= \begin{cases}\int_{\Omega}\left\{\epsilon|\nabla u|^{2}+\frac{W(u)}{\epsilon}\right\} \mathrm{d} x+\int_{\Omega} \frac{1}{\epsilon^{\alpha}} g\left(\frac{x}{\epsilon^{\alpha}}\right) u \mathrm{~d} x & \text { if } u \in H^{1}(\Omega), \\ & \text { otherwise. }\end{cases}
$$

We require that $g$ and $W$ satisfy the following assumptions:

(H1) $g \in L^{\infty}\left(\mathbb{R}^{N}\right)$ is a periodic function with cell domain $Q$, satisfying $\int_{Q} g \mathrm{~d} x=0$;

(H2) $W \in \operatorname{Lip}_{\text {loc }}(\mathbb{R}), W \geqslant 0, W(s)=0$ iff $s \in\{-1,1\}$ and $W(s)=W(-s)$;

(H3) There exist $\delta_{0} \in(0,1)$ and $C_{0}>0$ such that $W$ is strictly convex on the interval $\left(1-\delta_{0},+\infty\right)$ and

$$
\begin{array}{ll}
W(s) \leqslant C_{0}(s-1)^{2}, \quad & \forall s \in\left(1-\delta_{0}, 1+\delta_{0}\right), \\
W(s) \geqslant C_{0}^{-1}(s-1)^{2}, \quad \forall s \in\left(1-\delta_{0},+\infty\right)
\end{array}
$$

(H4) There exists $\rho>0$ such that

$$
W(1+s)-W(-1+s)=0 \quad \text { whenever } \quad|s|<\rho ;
$$

(H5) $g\left(x_{1}, \ldots, x_{i}, \ldots, x_{N}\right)=g\left(x_{1}, \ldots,-x_{i}, \ldots, x_{N}\right)$ for any $i \in\{1, \ldots, N\}$ (in this case we say that $g$ is symmetric).

A typical example of a function satisfying (H2) and (H3) but not (H4) is given by the doublewell potential defined by $W(s)=\left(1-s^{2}\right)^{2} / 2$. Assumption (H4) ensures that the two local minimizers around \pm 1 , i.e. the pure phases, have exactly the same energy (hence they are both global minimizers of the energy). Without that condition, the $\Gamma$-limit could become trivial (equal to 
0 or $+\infty)$. We observe that (H4) is not necessary in order to get the $\Gamma$-limit result when $\alpha<2 / 3$ (see Remark 4.10), whereas it is necessary if $\alpha>2 / 3$. Notice also that assumption (H3) implies

$$
\begin{array}{ll}
\left|W^{\prime}(x)\right| \geqslant C_{0}^{-1}|x-1| & \text { for } x \geqslant 1-\delta_{0}, \\
\left|W^{\prime}(x)\right| \geqslant C_{0}^{-1}|x+1| & \text { for } x \leqslant-1+\delta_{0} .
\end{array}
$$

We will see that in general $\lim _{\epsilon \rightarrow 0} \inf _{H^{1}(\Omega)} G_{\epsilon}(\cdot, \Omega)=-\infty$ for $\alpha>1 / 2$, hence we shall introduce an additive renormalization for the functionals. Let $\mathcal{R}_{\epsilon}$ be the family of all sets of the form $R=$ $\operatorname{int}\left(\bigcup_{z \in I} \epsilon^{\alpha}\{\bar{Q}+z\}\right)$, where $I$ is a finite subset of $\mathbb{Z}^{N}$. Given $\Omega \in \mathcal{A}$ and $u \in L^{1}(\Omega)$, we define the renormalized functionals as

$$
F_{\epsilon}(u, \Omega):= \begin{cases}\sup _{R \in \mathcal{R}_{\epsilon}, R \subseteq \Omega}\left\{G_{\epsilon}(u, R)-\inf _{H^{1}(R)} G_{\epsilon}(\cdot, R)\right\} & \text { if }\left\{R \in \mathcal{R}_{\epsilon}: R \subseteq \Omega\right\} \neq \emptyset, \\ 0 & \text { otherwise. }\end{cases}
$$

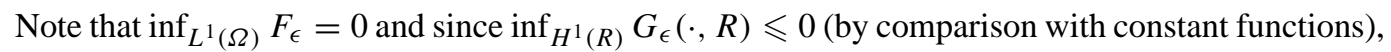
we also have $F_{\epsilon} \geqslant G_{\epsilon}$. Our main result is the following:

TheOREM 2.3 Let $0<\alpha<1$, let $W$ satisfy assumptions (H2) and (H3), and let $g$ satisfy (H1) and (H5). If $\alpha \geqslant 2 / 3$ we further assume (H4). Then there exists a constant $c_{0}=c_{0}(W)>0$ such that for any $g$ satisfying $\|g\|_{L^{N}} \leqslant c_{0}$, the $\Gamma$-limit (with respect to the $L^{1}$-topology) of $F_{\epsilon}(\cdot, \Omega$ ) exists for each $\Omega \in \mathcal{A}$ with Lipschitz boundary. Furthermore, we have

$$
\Gamma-\lim _{\epsilon \rightarrow 0} F_{\epsilon}(u, \Omega)= \begin{cases}\int_{\partial^{*} E \cap \Omega} \varphi\left(v_{E}\right) \mathrm{d} \mathcal{H}^{N-1} & \text { if } u=\chi_{E} \in B V(\Omega), \\ +\infty & \text { otherwise, }\end{cases}
$$

where $\varphi: \mathcal{S}^{N-1} \rightarrow(0, \infty)$, independent of $\alpha$, satisfies

$$
0<C \leqslant \varphi(v) \leqslant c_{W} \text { for all } v \in \mathcal{S}^{N-1},
$$

for some constant $C>0$, and its one-homogeneous extension

$$
\widetilde{\varphi}: \mathbb{R}^{N} \rightarrow[0, \infty), \quad x \mapsto \begin{cases}|x| \varphi(x /|x|) & \text { if } x \neq 0 \\ 0 & \text { if } x=0\end{cases}
$$

is convex.

REMARK 2.4 The function $\varphi$ can be computed as a limit of the averaged minimum energy on large boxes of the functional

$$
F_{g}^{A}\left(\chi_{E}\right):=c_{W} P(E, A)+\int_{A} g(x) \chi_{E}(x) \mathrm{d} x,
$$

defined for each Borel set $A \subset \Omega$ and each $\chi_{E} \in B V(\Omega)$ (see Theorem 5.9.

REMARK 2.5 We point out that the results of this section can be generalized to functionals with an $x$-dependence in the gradient term (see also [6]), like for example

$$
\widehat{G}_{\epsilon}(u, \Omega):=\int_{\Omega}\left\{\epsilon\left|A\left(\frac{x}{\epsilon^{\beta}}\right) \nabla u\right|^{2}+\frac{W(u)}{\epsilon}\right\} \mathrm{d} x+\int_{\Omega} \frac{1}{\epsilon^{\alpha}} g\left(\frac{x}{\epsilon^{\alpha}}\right) u \mathrm{~d} x,
$$

where $\alpha \in(0,1), \beta \geqslant 0$ and $A(x)$ is a positive definite symmetric matrix, periodically depending on $x$. 


\section{Estimates for the minimizers}

In the following, unless otherwise stated, we shall always take $\alpha \in(0,1)$. As we are interested in a local $\Gamma$-limit, we ultimately have to show that the renormalization is proportional to $|\Omega|$. This will be done by comparing with minimizers on a cube. We need the following definitions.

Definition 3.1 Let

$$
\widetilde{G}_{\epsilon}(u, \Omega):=\int_{\Omega}\left(\epsilon|\nabla u|^{2}+\frac{W(u)}{\epsilon}\right) \mathrm{d} x+\int_{\Omega} g u \mathrm{~d} x, \quad u \in H^{1}(\Omega) .
$$

Notice that, by the change of variables $y=\epsilon^{-\alpha} x$ and setting

$$
v(y)=u\left(\epsilon^{\alpha} y\right), \quad \Omega_{\epsilon}:=\left\{y \in \mathbb{R}^{N}: \epsilon^{\alpha} y \in \Omega\right\},
$$

for $\Omega \in \mathcal{R}_{\epsilon}$ we obtain the identity

$$
G_{\epsilon}(u, \Omega)=\epsilon^{\alpha(N-1)} \sum_{z \in \mathbb{Z}^{N}} \widetilde{G}_{\epsilon^{1-\alpha}}\left(v,(z+Q) \cap \epsilon^{-\alpha} \Omega\right) .
$$

Thanks to condition (H5), in order to study the structure of minimizers of $G_{\epsilon}$ on $R$, it is enough to analyze the minimizers on the cube with Neumann boundary conditions (which, again by condition (H5), are equivalent to periodic boundary conditions). Let us set

$$
c_{W}:=\int_{-1}^{1} \sqrt{W(t)} \mathrm{d} t, \quad \mathcal{B}:=\{u \in B V(Q): u(x) \in\{-1,1\} \text { a.e. }\},
$$

and consider the functional

$$
F_{g}^{Q}(u):= \begin{cases}c_{W} P(E, Q)+\int_{Q} g \chi_{E} \mathrm{~d} x & \text { if } u=\chi_{E} \in \mathcal{B}, \\ +\infty & \text { if } u \in L^{1}(Q) \backslash \mathcal{B} .\end{cases}
$$

From the result of [15, 16] we have $\Gamma-\lim \widetilde{G}_{\epsilon}(\cdot, Q)=F_{g}^{Q}$. This fact gives some hint on the asymptotic behavior of the minimizers of the functionals $\widetilde{G}_{\epsilon}(\cdot, Q)$. To see this, let us recall the following isoperimetric inequalities [11, Section 5.6].

Proposition 3.2 Let $\Omega \in \mathcal{A}$ with Lipschitz boundary. Then there exists a constant $I(\Omega)>0$ such that

1. $P(E, \Omega) \geqslant I(\Omega)(\min \{|E|,|\Omega \backslash E|\})^{(N-1) / N}$ for any $E \subseteq \Omega$;

2. $\int_{\Omega}|\nabla u| \geqslant 2 I(\Omega)\|u-\bar{u}\|_{N /(N-1)}$ for any $u \in B V(\Omega)$, where $\bar{u}:=|\Omega|^{-1} \int_{\Omega} u$.

Based on this result, we can derive:

Proposition 3.3 Let $\Omega \in \mathcal{A}$ with Lipschitz boundary. If $\|g\|_{L^{N}(Q)} \leqslant 2 c_{W} I(Q)$, then the minimizers of $F_{g}^{Q}$ are given by $u \equiv \pm 1$.

Proof. Since $F_{g}^{Q}(1)=F_{g}^{Q}(-1)=0$, it is enough to show $F_{g}^{Q}(u) \geqslant 0$ for all $u \in \mathcal{B}$. We have

$$
\begin{aligned}
c_{W} \int_{Q}|\nabla u| & \geqslant c_{W} 2 I(Q)\|u-\bar{u}\|_{N /(N-1)}, \\
\int_{Q} g u & =\int_{Q} g(u-\bar{u}) \geqslant-\|g\|_{N}\|u-\bar{u}\|_{N /(N-1)} .
\end{aligned}
$$


Thus,

$$
\begin{aligned}
F_{g}^{Q}(u) & \geqslant c_{W} 2 I(Q)\|u-\bar{u}\|_{N /(N-1)}-\|g\|_{N}\|u-\bar{u}\|_{N /(N-1)}, \\
& =\|u-\bar{u}\|_{N /(N-1)}\left(c_{W} 2 I(Q)-\|g\|_{N}\right),
\end{aligned}
$$

and the last term is nonnegative by assumption.

Proposition 3.3 implies that if the minimizers of $\widetilde{G}_{\epsilon}(\cdot, Q)$ exist and converge in $L^{1}$, they must converge to \pm 1 . We need now to quantify this information, i.e. to obtain rates in $\epsilon$.

Proposition 3.4 Assume (H1) to (H3). Then for any $u \in H^{1}(\Omega)$ we have

$$
\widetilde{G}_{\epsilon}(t \wedge u \vee(-t), \Omega)<\widetilde{G}_{\epsilon}(u, \Omega) \quad \forall t>1+\epsilon C_{0}\|g\|_{\infty} .
$$

Proof. By setting $\Omega_{t}:=\{|u|>t\}$, from (H2) and 2.2), we get

$$
\begin{aligned}
\widetilde{G}_{\epsilon}(u, \Omega)-\widetilde{G}_{\epsilon}(t \wedge u \vee(-t), \Omega) & \geqslant \frac{1}{\epsilon} \int_{\Omega_{t}}(W(u)-W(t)) \mathrm{d} x+\int_{\Omega_{t}} g(u-\operatorname{sgn}(u) t) \mathrm{d} x, \\
& \geqslant \frac{1}{\epsilon} \int_{\Omega_{t}}\left(W^{\prime}(t)-\epsilon\|g\|_{\infty}\right)(|u|-t) \mathrm{d} x, \\
& \geqslant \frac{1}{\epsilon} \int_{\Omega_{t}}\left(C_{0}^{-1}(t-1)-\epsilon\|g\|_{\infty}\right)(|u|-t) \mathrm{d} x,
\end{aligned}
$$

and the last expression is positive whenever $t>1+\epsilon C_{0}\|g\|_{\infty}$.

The following definition introduces a cutting and reflection procedure, which gives a function $u^{t}$ assuming values only in one of the convex regions of the potential $W$.

DEFINITION 3.5 Given $u \in H^{1}(\Omega)$ and $t>0$, we define

$$
u^{t}:= \begin{cases}|u| \vee t & \text { if }|\{u>0\}| \geqslant \frac{1}{2}|\Omega|, \\ -(|u| \vee t) & \text { if }|\{u>0\}|<\frac{1}{2}|\Omega| .\end{cases}
$$

We are going to use this cutting procedure to give an estimate of the energy required to have a sign change of the function $u$.

Proposition 3.6 Let $\Omega \in \mathcal{A}$ with Lipschitz boundary. Assume (H1) to (H3) and $\epsilon\|g\|_{\infty}<$ $\frac{1}{2} C_{0}^{-1} \delta_{0}$. Then there exist a constant $t_{0}$ with $\max \left\{\frac{1}{2}, 1-\delta_{0}\right\}<t_{0}<1$ and $\omega_{0}>0\left(t_{0}, \omega_{0}\right.$ depending only on $W$ ) such that

$$
\widetilde{G}_{\epsilon}(u, \Omega)-\widetilde{G}_{\epsilon}\left(u^{t}, \Omega\right) \geqslant\left(\omega_{0}-\frac{8}{t_{0}} \frac{\|g\|_{L^{N}}}{I(\Omega)}\right) \int_{-t / 2}^{t / 2} P(\{u<s\}, \Omega) \mathrm{d} s
$$

whenever $u \in H^{1}(\Omega)$ and $t \in\left(t_{0}, 1-2 \epsilon C_{0}\|g\|_{\infty}\right)$. Moreover, the inequality is strict if $|\{|u|<t\}|$ $>0$.

Proof. Assume without loss of generality that $|\{u>0\}| \geqslant|\Omega| / 2$ and, in the light of Proposition 3.4. that $|u| \leqslant 2-t$. Recall that $W(u)=W(-u)$ and compute

$$
\begin{aligned}
\widetilde{G}_{\epsilon}(u, \Omega)-\widetilde{G}_{\epsilon}\left(u^{t}, \Omega\right)= & \int_{\{-t<u<t\}}\left\{\epsilon|\nabla u|^{2}+\frac{W(u)-W(t)}{\epsilon}+g(u-t)\right\} \mathrm{d} x \\
& +2 \int_{\{u \leqslant-t\}} g u \mathrm{~d} x=G_{1}+G_{2}+G_{3},
\end{aligned}
$$


where

$$
\begin{aligned}
G_{1} & :=\int_{\{-t \leqslant u<t\}}\left(\epsilon|\nabla u|^{2}+\frac{W(u)-W(t)}{2 \epsilon}\right) \mathrm{d} x, \\
G_{2} & :=\int_{\{-t<u<t\}} \frac{W(u)-W(t)}{2 \epsilon} \mathrm{d} x+\int_{\{-t / 2 \leqslant u<t\}} g(u-t) \mathrm{d} x, \\
G_{3} & :=\int_{\{-t<u<-t / 2\}} g(u-t) \mathrm{d} x+2 \int_{\{u \leqslant-t\}} g u \mathrm{~d} x .
\end{aligned}
$$

Let us first observe that $(\mathrm{H} 2)$ and $(\mathrm{H} 3)$ imply the existence of a value $t_{0}$ (depending only on $W$ ) with $\max \left\{1 / 2,1-\delta_{0}\right\}<t_{0}<1$ such that, for all $t \in\left(t_{0}, 1\right)$, we have

$$
\begin{gathered}
W(s) \geqslant W(t)+W^{\prime}(t)(s-t) \quad \forall s>-1 / 2, \\
W(s)-W(t) \geqslant 0 \quad \forall|s|<t \quad \text { and } \quad \inf _{|s|<1 / 2}\left\{W(s)-W\left(t_{0}\right)\right\}>0 .
\end{gathered}
$$

Let us also define $\omega_{0}:=\inf _{|s|<1 / 2} \sqrt{2\left\{W(s)-W\left(t_{0}\right)\right\}}$.

1. By using the Schwarz inequality and co-area formula, we estimate $G_{1}$ as follows:

$$
G_{1} \geqslant \int_{\{-t \leqslant u<t\}} \sqrt{2\{W(u)-W(t)\}}|\nabla u| \mathrm{d} x \geqslant \omega_{0} \int_{-t / 2}^{t / 2} P(\{u<s\}, \Omega) \mathrm{d} s,
$$

since $\inf _{|s|<t / 2} \sqrt{2\{W(s)-W(t)\}} \geqslant \inf _{|s|<1 / 2} \sqrt{2\left\{W(s)-W\left(t_{0}\right)\right\}}=\omega_{0}$.

2. We show that $G_{2} \geqslant 0$. Using [3.5), for all $t_{0}<t<1-2 \epsilon C_{0}\|g\|_{\infty}$ we get

$$
\begin{aligned}
G_{2} & \geqslant \int_{\{-t / 2 \leqslant u<t\}}\left(\frac{W(u)-W(t)}{2 \epsilon}+g(u-t)\right) \mathrm{d} x \\
& \geqslant \int_{\{-t / 2 \leqslant u<t\}} \frac{-W^{\prime}(t)-2 \epsilon g}{2 \epsilon}(t-u) \mathrm{d} x \\
& \geqslant \int_{\{-t / 2 \leqslant u<t\}} \frac{C_{0}^{-1}(1-t)-2 \epsilon\|g\|_{\infty}}{2 \epsilon}(t-u) \mathrm{d} x \geqslant 0
\end{aligned}
$$

and $G_{2}>0$ if $|\{u<t\}|>0$.

3. In order to estimate $G_{3}$, we use $|u| \leqslant 2-t$ and the Hölder inequality to get

$$
\begin{aligned}
\left|G_{3}\right| & \leqslant 2 t \int_{\{-t<u<-t / 2\}}|g| \mathrm{d} x+2(2-t) \int_{\{u<-t\}}|g| \mathrm{d} x \\
& \leqslant 4 \int_{\{u<-t / 2\}}|g| \mathrm{d} x \leqslant 4\|g\|_{L^{N}}|\{u<-t / 2\}|^{(N-1) / N} .
\end{aligned}
$$

From the fact that $|\{u<s\}|$ is a nondecreasing function of $s$, and using Proposition 3.2 together with the assumption $|\{u>0\}| \geqslant|\Omega| / 2$, we get

$$
\frac{t}{2}|\{u<-t / 2\}|^{(N-1) / N} \leqslant \int_{-t / 2}^{0}|\{u<s\}|^{(N-1) / N} \mathrm{~d} s \leqslant \frac{1}{I(\Omega)} \int_{-t / 2}^{0} P(\{u<s\}, \Omega) \mathrm{d} s .
$$


Therefore, 3.9) gives

$$
\left|G_{3}\right| \leqslant \frac{8}{t} \frac{\|g\|_{L^{N}}}{I(\Omega)} \int_{-t / 2}^{0} P(\{u<s\}, \Omega) \mathrm{d} s \leqslant \frac{8}{t_{0}} \frac{\|g\|_{L^{N}}}{I(\Omega)} \int_{-t / 2}^{0} P(\{u<s\}, \Omega) \mathrm{d} s .
$$

4. Finally, from 3.7$), 3.8$ and 3.10 we obtain

$$
G_{1}+G_{2}+G_{3} \geqslant\left(\omega_{0}-\frac{8}{t_{0}} \frac{\|g\|_{L^{N}}}{I(\Omega)}\right) \int_{-t / 2}^{t / 2} P(\{u<s\}, \Omega) \mathrm{d} s .
$$

Moreover (3.8) implies that the inequality is strict if $|\{u<t\}|>0$.

In the following proposition, we show that the functional $\widetilde{G}_{\epsilon}$ admits global minimizers which are close to +1 or -1 of an order $\epsilon$ (see [13] for a similar result in the case of minimizers of (1.1) with a volume constraint).

Proposition 3.7 Let $\Omega \in \mathcal{A}$ with Lipschitz boundary. Assume (H1) to (H3) and $\epsilon\|g\|_{\infty}<$ $(1 / 2) C_{0}^{-1} \delta_{0}$. Then:

1. The functional $\sqrt{3.1}$ admits a global minimizer $u_{\epsilon}$ in $H^{1}(\Omega)$.

2. Let $H_{ \pm}^{1}(\Omega):=\left\{u \in H^{1}(\Omega): \pm u \geqslant 0\right.$ a.e. in $\left.\Omega\right\}$. Then there exist positive constants $c_{0}(\Omega, W)$, $C_{1}(\Omega, W)$ and $\epsilon_{0}(\Omega, W)$ such that for $\|g\|_{L^{N}} \leqslant c_{0}$ any global minimizer $u_{\epsilon}$ must be contained in $H_{+}^{1}$ or $H_{-}^{1}$. Moreover, any minimizer $u_{\epsilon}^{ \pm} \in H_{ \pm}^{1}$ has the following property:

$$
\left\|u_{\epsilon}^{+}-1\right\|_{\infty} \leqslant C_{1} \epsilon, \quad\left\|u_{\epsilon}^{-}+1\right\|_{\infty} \leqslant C_{1} \epsilon \quad \text { for } \epsilon<\epsilon_{0} .
$$

Since the restriction of $\widetilde{G}_{\epsilon}(\cdot, \Omega)$ to $B_{\delta_{0}}^{\|\cdot\|_{\infty}}(+1)$ (respectively to $B_{\delta_{0}}^{\|\cdot\|_{\infty}}(-1)$ ) is convex, Proposition 3.7 implies

Corollary 3.8 Let $\Omega \in \mathcal{A}$ with Lipschitz boundary. Assume (H1) to (H3), and $\|g\|_{L^{N}} \leqslant$ $c_{0}(W, \Omega)$. Then for any $\epsilon$ such that $\epsilon\|g\|_{\infty}<C_{0}^{-1} \delta_{0}$, the functional $\widetilde{G}_{\epsilon}(\cdot, \Omega)$ has exactly one absolute minimizer $u_{\epsilon}^{+}$in $H_{+}^{1}(\Omega)$ and one absolute minimizer $u_{\epsilon}^{-}$in $H_{-}^{1}(\Omega)$. Moreover, there exists $t_{0} \in\left(1-\delta_{0}, 1\right)$ such that for all $u \in H^{1}(\Omega)$ we have

$$
\widetilde{G}_{\epsilon}(u, \Omega)-\min \left(\widetilde{G}_{\epsilon}\left(u_{\epsilon}^{+}, \Omega\right), \widetilde{G}_{\epsilon}\left(u_{\epsilon}^{-}, \Omega\right)\right) \geqslant C \int_{-t_{0} / 2}^{t_{0} / 2} P(\{u<s\}, \Omega) \mathrm{d} s .
$$

If $W$ satisfies (H4), we also have $u_{\epsilon}^{+}=2+u_{\epsilon}^{-}$and $\widetilde{G}_{\epsilon}\left(u_{\epsilon}^{+}, \Omega\right)=\widetilde{G}_{\epsilon}\left(u_{\epsilon}^{-}, \Omega\right)$, and $u_{\epsilon}^{ \pm}$are the only global minimizers of $\widetilde{G}_{\epsilon}$ on $H^{1}(\Omega)$.

Proof of Proposition 3.7 The existence of a global minimizer follows from classical results (see for example [9, Theorem 2.6]). From Proposition 3.4 we see immediately that the global minimizer $u_{\epsilon}$ fulfills $u_{\epsilon} \leqslant 1+C \epsilon$ or $u_{\epsilon} \geqslant-1-C \epsilon$ for some $C$ depending only on $\Omega$ and $W$.

Assume now without loss of generality that $\left|\left\{u_{\epsilon}>0\right\}\right| \geqslant|\Omega| / 2$. Proposition 3.6 tells us that for a minimizer there exists a $t$ with $1-\delta_{0}<t<1$ such that $\left|\left\{-t / 2<u_{\epsilon}<t\right\}\right|=0$. Moreover it implies that $P\left(\left\{u_{\epsilon}<s\right\}, \Omega\right)=0$ for some $s \in(-t / 2, t / 2)$. Hence the isoperimetric inequality implies that also $\left|\left\{u_{\epsilon}<-t / 2\right\}\right|=0$ is empty. Therefore $u_{\epsilon}(x) \in\left(1-\delta_{0}, 1+\delta_{0}\right)$ almost everywhere. 
Proposition 3.9 Assume (H1) to $(\mathrm{H} 3)$ with $g \not \equiv 0$. Then

$$
0>\min _{H^{1}(Q)}\left\{\widetilde{G}_{\epsilon}(\cdot, Q)\right\} \geqslant-2 C_{0}\|g\|_{\infty}^{2} \epsilon .
$$

Moreover, let $\Omega \in \mathcal{A}$. Then, for any $(\epsilon, \alpha)$ and any $R_{\epsilon} \in \mathcal{R}_{\epsilon}$ with $\mathcal{R}_{\epsilon} \subset \Omega$, we have

$$
0>\min _{H^{1}\left(R_{\epsilon}\right)}\left\{G_{\epsilon}\left(\cdot, R_{\epsilon}\right)\right\} \geqslant-2|\Omega| C_{0}\|g\|_{\infty}^{2} \epsilon^{1-2 \alpha} .
$$

In particular, as $\epsilon \rightarrow 0$,

$$
\min _{H^{1}\left(R_{\epsilon}\right)}\left\{G_{\epsilon}\left(\cdot, R_{\epsilon}\right)\right\}= \begin{cases}o(1) & \text { if } \alpha \in(0,1 / 2), \\ O(1) & \text { if } \alpha=1 / 2 .\end{cases}
$$

If $\alpha>1 / 2$, there exists $R_{\epsilon} \in \mathcal{R}_{\epsilon}, R_{\epsilon} \subset \Omega$, such that

$$
\lim _{\epsilon \rightarrow 0} \min _{H^{1}\left(R_{\epsilon}\right)}\left\{G_{\epsilon}\left(\cdot, R_{\epsilon}\right)\right\}=-\infty .
$$

Proof. Let $v$ be a positive global minimizer of $\widetilde{G}_{\epsilon}$ on $H^{1}(Q)$. By Propositions 3.4 and 3.7 we know that $\|v-1\|_{\infty} \leqslant 2 C_{0}\|g\|_{\infty} \epsilon$. This estimate, together with the assumption that $g$ is of average zero on $Q$, yields

$$
\widetilde{G}_{\epsilon}(v, Q) \geqslant \int_{Q} g v \mathrm{~d} y \geqslant-\|g\|_{\infty}\|v-1\|_{\infty} \geqslant-2 C_{0}\|g\|_{\infty}^{2} \epsilon .
$$

This proves (3.12). Now, note that the number of cubes of size $\epsilon^{\alpha}$ contained in $R_{\epsilon}$ is equal to $\left|R_{\epsilon}\right| / \epsilon^{\alpha N}$. Hence, by using 3.2, for each $u \in H^{1}\left(R_{\epsilon}\right)$ we get

$$
G_{\epsilon}\left(u, R_{\epsilon}\right) \geqslant \frac{\left|R_{\epsilon}\right|}{\epsilon^{\alpha N}} \epsilon^{\alpha(N-1)} \min _{H^{1}(Q)} \widetilde{G}_{\epsilon^{1-\alpha}}(\cdot, Q)=\frac{\left|R_{\epsilon}\right|}{\epsilon^{\alpha}} \min _{H^{1}(Q)} \widetilde{G}_{\epsilon^{1-\alpha}}(\cdot, Q) .
$$

Hence, from 3.15, 3.12) and the fact that $\left|R_{\epsilon}\right| \leqslant|\Omega|$, we derive (3.13).

Consider now the case $\alpha>1 / 2$. Choose a function $v \in C_{c}^{1}(Q)$ such that $\int_{Q} g v \mathrm{~d} x<0$ (which is always possible if $g \not \equiv 0$ ) and extend it periodically on $\mathbb{R}^{N}$. Consider $R_{\epsilon} \in \mathcal{R}_{\epsilon}$ with $\left|R_{\epsilon}\right| \geqslant|\Omega| / 2$. Then, using 3.2 as before, we get

$$
\begin{aligned}
G_{\epsilon}\left(1+\epsilon^{1 / 2} v\left(\frac{x}{\epsilon^{\alpha}}\right), R_{\epsilon}\right) & =\frac{\left|R_{\epsilon}\right|}{\epsilon^{\alpha}} \widetilde{G}_{\epsilon^{1-\alpha}}\left(1+\epsilon^{1 / 2} v, Q\right) \\
& \leqslant \frac{|\Omega|}{2} \int_{Q}\left(\epsilon^{2(1-\alpha)}|\nabla v|^{2}+C_{0} v^{2}+\epsilon^{1 / 2-\alpha} g v\right) \mathrm{d} x \\
& \rightarrow-\infty \text { as } \epsilon \rightarrow 0 .
\end{aligned}
$$

The previous proposition shows that $F_{\epsilon}$ and $G_{\epsilon}$ have the same $\Gamma$-limit whenever $\alpha<1 / 2$ and so the renormalization is not needed in this case, whereas the functionals $G_{\epsilon}$ typically converge to $-\infty$ when $\alpha>1 / 2$. We give the following definition in order to express the additive renormalization in a more convenient way.

DEFINITION 3.10

1. Let $u_{\epsilon^{1-\alpha}}^{ \pm}$denote the minimizer of $\widetilde{G}_{\epsilon^{1-\alpha}}$ on $H^{1}(Q) \cap\{ \pm u \geqslant 0\}$.

2. Let $c_{\epsilon}:=\epsilon^{-\alpha} \inf _{v \in H^{1}(Q)} \widetilde{G}_{\epsilon^{1-\alpha}}(v, Q)$. 
Proposition 3.11 Assume (H1) to (H3). If furthermore (H5) holds, i.e. if $g$ is symmetric, then the functions which minimize $\min _{H^{1}(Q)} \widetilde{G}_{\epsilon}(\cdot, Q)$ are periodic. Moreover, if (H4) holds then

$$
\min _{H^{1}(R)} G_{\epsilon}(\cdot, R)=\frac{|R|}{\epsilon^{\alpha}} \widetilde{G}_{\epsilon^{1-\alpha}}\left(u_{\epsilon^{1-\alpha}}^{ \pm}, Q\right)=|R| c_{\epsilon} .
$$

Moreover, the functional $F_{\epsilon}$ is additive on disjoint sets contained in $\mathcal{R}_{\epsilon}$.

Proof. Denote by $H_{p}^{1}(Q)$ the class of periodic $H^{1}$-functions on the unit cube.

Recall that the minimizers $u^{+}$(resp. $u^{-}$) are unique in the class of positive (resp. negative) $H^{1}$ functions. By symmetry of $g, u^{+}\left(x_{1}, \ldots,-x_{i}, \ldots x_{n}\right)$ is also a minimizer and thus equal to $u^{+}$. The same holds for $u^{-}$. In particular the traces of $u^{ \pm}$on opposite facets of the cube coincide, so $u^{ \pm} \in H_{p}^{1}(Q)$.

\section{4. $\Gamma$-convergence}

In this section, we establish the $\Gamma$-convergence of the functionals $F_{\epsilon}$ for $\epsilon \rightarrow 0$. In order to proceed, we need to distinguish between cubes in which a function $u$ is mostly positive and those in which $u$ is mostly negative.

Definition 4.1 Given $\left(R_{\epsilon}, u\right) \in \mathcal{R}_{\epsilon} \times H^{1}\left(R_{\epsilon}\right)$, we define

$$
\begin{aligned}
& Z_{\epsilon}^{+}:=\left\{z \in \mathbb{Z}^{N}: \epsilon^{\alpha}(Q+z) \subset R_{\epsilon},\left|\left\{u_{\epsilon}>0\right\} \cap \epsilon^{\alpha}(Q+z)\right| \geqslant \frac{1}{2}\left|\epsilon^{\alpha}(Q+z)\right|\right\}, \\
& Z_{\epsilon}^{-}:=\left\{z \in \mathbb{Z}^{N}: \epsilon^{\alpha}(Q+z) \subset R_{\epsilon},\left|\left\{u_{\epsilon}>0\right\} \cap \epsilon^{\alpha}(Q+z)\right|<\frac{1}{2}\left|\epsilon^{\alpha}(Q+z)\right|\right\}, \\
& R_{\epsilon}^{ \pm}:=\bigcup_{z \in Z_{\epsilon}^{ \pm}} \epsilon^{\alpha}(Q+z) .
\end{aligned}
$$

Using the notation introduced in the above definition, we show:

LEMmA 4.2 There exists $C>0$ such that for any $\left(R_{\epsilon}, u\right) \in \mathcal{R}_{\epsilon} \times H^{1}\left(R_{\epsilon}\right)$, the following holds:

$$
\begin{gathered}
\left|\{u \leqslant-1 / 2\} \cap R_{\epsilon}^{+}\right|+\left|\{u \geqslant 1 / 2\} \cap R_{\epsilon}^{-}\right| \leqslant C \epsilon^{\alpha} F_{\epsilon}\left(u, R_{\epsilon}\right), \\
\int_{R_{\epsilon}}\left\{\frac{W(u)}{\epsilon}+\frac{u}{\epsilon^{\alpha}} g\left(\frac{x}{\epsilon^{\alpha}}\right)\right\} \mathrm{d} x \geqslant-C\left\{F_{\epsilon}\left(u, R_{\epsilon}\right)+\left|R_{\epsilon}\right| \epsilon^{1-2 \alpha}\right\}, \\
\int_{R_{\epsilon}} \frac{W(u)}{\epsilon} \mathrm{d} x \leqslant C\left\{F_{\epsilon}\left(u, R_{\epsilon}\right)+\left|R_{\epsilon}\right| \epsilon^{1-2 \alpha}\right\} .
\end{gathered}
$$

Proof. We first show 4.1). By setting $v(x)=u\left(\epsilon^{-\alpha} x\right)$, we have

$$
F_{\epsilon}\left(u, R_{\epsilon}^{+}\right) \geqslant \epsilon^{(N-1) \alpha} \sum_{z \in Z_{\epsilon}^{+}}\left\{\widetilde{G}_{\epsilon^{1-\alpha}}(v, z+Q)-\widetilde{G}_{\epsilon^{1-\alpha}}\left(u^{+}, z+Q\right)\right\} .
$$

Lemma 3.8 and the isoperimetric inequality applied to 4.4 yield

$$
F_{\epsilon}\left(u, R_{\epsilon}^{+}\right) \geqslant C \epsilon^{(N-1) \alpha} \sum_{z \in Z_{\epsilon}^{+}}|\{v \leqslant-1 / 2\} \cap(z+Q)|^{(N-1) / N} .
$$


Using, in the relation above, the inequality

$$
\sum_{i=1}^{m}\left|A_{i}\right| \leqslant \max _{i \in\{1, \ldots, m\}}\left\{\left|A_{i}\right|^{1 / N}\right\} \sum_{i=1}^{m}\left|A_{i}\right|^{(N-1) / N}
$$

(holding for any $m \in \mathbb{N}$ and any $A_{1}, \ldots, A_{m} \in \mathbb{R}$ ), we derive

$$
F_{\epsilon}\left(u, R_{\epsilon}^{+}\right) \geqslant C \epsilon^{(N-1) \alpha} \sum_{z \in Z_{\epsilon}^{+}}|\{v \leqslant-1 / 2\} \cap(z+Q)|=C \epsilon^{-\alpha}\left|\{u \leqslant-1 / 2\} \cap R_{\epsilon}^{+}\right| .
$$

Hence, arguing in the same way on $R_{\epsilon}^{-}$, we finally derive

$$
\epsilon^{\alpha} F_{\epsilon}\left(u, R_{\epsilon}^{ \pm}\right) \geqslant C\left|\{u \leqslant-1 / 2\} \cap R_{\epsilon}^{ \pm}\right| .
$$

Now, (4.6) together with $F_{\epsilon}\left(u, R_{\epsilon}\right) \geqslant F_{\epsilon}\left(u, R_{\epsilon}^{+}\right)+F_{\epsilon}\left(u, R_{\epsilon}^{-}\right)$implies 4.1 .

To prove (4.2) and (4.3), we will show

$$
\int_{R_{\epsilon}}\left\{\frac{W(u)}{2 \epsilon}+\frac{u}{\epsilon^{\alpha}} g\left(\frac{x}{\epsilon^{\alpha}}\right)\right\} \mathrm{d} x \geqslant-C\left\{F_{\epsilon}\left(u, R_{\epsilon}\right)+\left|R_{\epsilon}\right| \epsilon^{1-2 \alpha}\right\} .
$$

First let us introduce the notation

$$
B_{\epsilon}^{ \pm}:=\left\{x \in R_{\epsilon}^{ \pm}: \pm u_{\epsilon}(x)<-1 / 2\right\} .
$$

We note that by $(\mathrm{H} 2)$ and $(\mathrm{H} 3)$ we can find a constant $c$ with $0<c<C_{0}^{-1}$ such that $W(u) \geqslant$ $c(u-1)^{2}$ for $u \in[-1 / 2, \infty)$. Moreover, there exist $C, \epsilon_{0}>0$ such that

$$
\frac{W(u)}{2}+\epsilon^{1-\alpha}(u-1) g\left(\frac{x}{\epsilon^{\alpha}}\right)>0 \quad \text { for }|u|>C, \epsilon<\epsilon_{0} .
$$

Hence

$$
\begin{aligned}
\int_{R_{\epsilon}^{+}}\left\{\frac{W(u)}{2 \epsilon}\right. & \left.+\frac{u}{\epsilon^{\alpha}} g\left(\frac{x}{\epsilon^{\alpha}}\right)\right\} \mathrm{d} x=\int_{R_{\epsilon}^{+}}\left\{\frac{W(u)}{2 \epsilon}+\frac{u-1}{\epsilon^{\alpha}} g\left(\frac{x}{\epsilon^{\alpha}}\right)\right\} \mathrm{d} x \\
& =\int_{R_{\epsilon}^{+} \backslash B_{\epsilon}^{+}}\left\{\frac{W(u)}{2 \epsilon}+\frac{u-1}{\epsilon^{\alpha}} g\left(\frac{x}{\epsilon^{\alpha}}\right)\right\} \mathrm{d} x+\int_{B_{\epsilon}^{+}}\left\{\frac{W(u)}{2 \epsilon}+\frac{u-1}{\epsilon^{\alpha}} g\left(\frac{x}{\epsilon^{\alpha}}\right)\right\} \mathrm{d} x \\
& \geqslant \int_{R_{\epsilon}^{+} \backslash B_{\epsilon}^{+}}\left\{\frac{c(u-1)^{2}}{\epsilon}+\frac{u-1}{\epsilon^{\alpha}} g\left(\frac{x}{\epsilon^{\alpha}}\right)\right\} \mathrm{d} x-\frac{C}{\epsilon^{\alpha}}\|g\|_{\infty}\left|B_{\epsilon}^{+}\right| \\
& \geqslant-\frac{1}{\epsilon}\left|R_{\epsilon}^{+}\right| \frac{\left(\epsilon^{1-\alpha}\|g\|_{\infty}\right)^{2}}{4 c}-C\|g\|_{\infty} \epsilon^{\alpha} F_{\epsilon}\left(u, R_{\epsilon}\right) \epsilon^{-\alpha} \\
& \geqslant-C^{\prime}\left\{F_{\epsilon}\left(u, R_{\epsilon}^{+}\right)+\left|R_{\epsilon}^{+}\right| \epsilon^{1-2 \alpha}\right\} .
\end{aligned}
$$

The corresponding estimate holds for $R_{\epsilon}^{-}$as well and so we get 4.7 .

From (4.7), we derive immediately 4.2$]$. Furthermore, since the renormalization per unit volume $c_{\epsilon}$ is negative, using (4.7) we can estimate

$$
\begin{aligned}
\frac{1}{2} \int_{R_{\epsilon}} \frac{W(u)}{\epsilon} \mathrm{d} x & \leqslant F_{\epsilon}\left(u, R_{\epsilon}\right)-\int_{R_{\epsilon}}\left\{\frac{W(u)}{2 \epsilon}+\frac{u}{\epsilon^{\alpha}} g\left(\frac{x}{\epsilon^{\alpha}}\right)\right\} \mathrm{d} x \\
& \leqslant C\left\{F_{\epsilon}\left(u, R_{\epsilon}\right)+\left|R_{\epsilon}\right| \epsilon^{1-2 \alpha}\right\} .
\end{aligned}
$$


As a first step we show that the $\Gamma$-limit (if it exists) concentrates exactly on the class of characteristic functions of sets of finite perimeter.

Proposition 4.3 Let $\Omega \in \mathcal{A}$ and $u_{\epsilon} \in L^{1}(\Omega)$ be such that $\lim _{\sup } \sup _{\epsilon \rightarrow 0} F_{\epsilon}\left(u_{\epsilon}\right)<\infty$. Then:

(a) If $u_{\epsilon_{n}} \rightarrow u$ in $L^{1}(\Omega)$ for any subsequence $\epsilon_{n} \rightarrow 0$, then $|u|=1$ a.e. in $\Omega$;

(b) There exists a subsequence $\epsilon_{n} \rightarrow 0$ and $u \in B V(\Omega)$ with $|u|=1$ a.e. in $\Omega$ such that $\left\|u_{\epsilon_{n}}-u\right\|_{L_{\mathrm{loc}}^{1}(\Omega)} \rightarrow 0$. Moreover, there exists $C:=C(W, g)>0$ such that

$$
\int_{\Omega}|\nabla u| \leqslant C \liminf _{\epsilon_{n} \rightarrow 0} F_{\epsilon_{n}}\left(u_{\epsilon_{n}}, \Omega\right) .
$$

Proof. Let $R_{\epsilon} \in \mathcal{R}_{\epsilon}$ be such that $F_{\epsilon}\left(u_{\epsilon}, \Omega\right)=F_{\epsilon}\left(u_{\epsilon}, R_{\epsilon}\right)$.

(a) From Lusin's and Egoroff's Theorems (see [11]), we deduce the existence of a compact set $K \subset \Omega$ such that (up to a subsequence)

$$
|K| \neq 0,\left.\quad u\right|_{K} \text { continuous, } \quad u_{n} \rightarrow u \text { in } L^{\infty}(K) .
$$

Since $|u| \not \equiv 1$ we can further assume the existence of a constant $\eta>0$ such that

$$
|| u_{n}(x)|-1| \geqslant \eta>0 \quad \forall x \in K, n \in \mathbb{N} .
$$

Letting now $c:=\min _{|| s|-1| \geqslant \eta} W(s)>0$, for $n$ large enough we have

$$
\begin{aligned}
F_{\epsilon_{n}}\left(u_{n}, \Omega\right) & \geqslant G_{\epsilon_{n}}\left(u_{n}\right) \geqslant \int_{K} \frac{W\left(u_{n}\right)}{\epsilon_{n}}+\frac{1}{\epsilon_{n}^{\alpha}} \int_{\Omega} g\left(\frac{x}{\epsilon_{n}^{\alpha}}\right) u_{n} \\
& \geqslant c \frac{|K|}{\epsilon_{n}}-\frac{\|g\|_{\infty}}{\epsilon_{n}^{\alpha}} \int_{\Omega}|u| \rightarrow+\infty .
\end{aligned}
$$

(b) By referring to Definition 4.1 we set

$$
\sigma\left(u_{\epsilon}, z\right)=\left\{\begin{array}{ll}
1 & \text { if } z \in Z_{\epsilon}^{+}, \\
-1 & \text { if } z \in Z_{\epsilon}^{-},
\end{array} \quad\left[H u_{\epsilon}\right](x)= \begin{cases}1 & \text { if } x \in R_{\epsilon}^{+}, \\
-1 & \text { if } x \in R_{\epsilon}^{-} .\end{cases}\right.
$$

We shall show that

$$
\left\|u_{\epsilon}-H u_{\epsilon}\right\|_{L^{1}\left(R_{\epsilon}\right)} \rightarrow 0(\text { as } \epsilon \rightarrow 0) \text { and }\left\|H u_{\epsilon}\right\|_{B V\left(R_{\epsilon}\right)} \leqslant C .
$$

Set

$$
B_{\epsilon, \delta}:=\left\{x \in R_{\epsilon}:\left|u_{\epsilon}(x)\right|<1-\delta\right\} \quad(\delta>0) .
$$

Note that for $0<\delta \ll 1$,

$$
\left\|u_{\epsilon}-H u_{\epsilon}\right\|_{L^{1}\left(R_{\epsilon}\right)} \leqslant \delta\left|R_{\epsilon}\right|+3\left(\left|B_{\epsilon}^{+}\right|+\left|B_{\epsilon}^{-}\right|\right)+2\left|B_{\epsilon, \delta}\right|+2 \int_{\left\{\left|u_{\epsilon}\right|>1+\delta\right\}}\left|u_{\epsilon}\right| \mathrm{d} x .
$$

By applying Lemma 4.2, we get $\left|B_{\epsilon}^{+}\right|+\left|B_{\epsilon}^{-}\right| \leqslant C \epsilon^{\alpha}$ and so $\left|B_{\epsilon}^{+}\right|+\left|B_{\epsilon}^{-}\right| \rightarrow 0$. By (H2), (H3) and the bound on the energy

$$
\lim _{\epsilon \rightarrow 0}\left(\left|B_{\epsilon, \delta}\right|+\int_{\left\{\left|u_{\epsilon}\right|>1+\delta\right\}}\left|u_{\epsilon}\right| \mathrm{d} x\right)=0
$$


we then obtain the first statement in 4.12 . To prove the second one, we note that, by construction, the total variation of $H u_{\epsilon}$ can be estimated by

$$
\int_{R_{\epsilon}}\left|\nabla\left[H u_{\epsilon}\right]\right| \leqslant \frac{\epsilon^{(N-1) \alpha}}{4} \sum_{\left|z_{i}-z_{j}\right|=1}\left|\sigma_{\epsilon}\left(z_{i}\right)-\sigma_{\epsilon}\left(z_{j}\right)\right|^{2} .
$$

Now consider a pair of cubes $Q_{i}:=\epsilon^{\alpha}\left(z_{i}+Q\right)(i=1,2)$ such that $\left(z_{1}, z_{2}\right) \in Z_{\epsilon}^{+} \times Z_{\epsilon}^{-}$and $\left|z_{1}-z_{2}\right|=1$ (i.e. the cubes are adjacent). By setting $\mathcal{C}:=\operatorname{int}\left(\bar{Q}_{1} \cup \bar{Q}_{2}\right)$, we claim that there exists $C>0$ such that

$$
F_{\epsilon}\left(u_{\epsilon}, \mathcal{C}\right) \geqslant C \epsilon^{(N-1) \alpha} \text {. }
$$

Case 1: $\left|Q_{1} \cap\left\{0<u_{\epsilon}<1 / 2\right\}\right|>\left|Q_{1}\right| / 4$ or $\left|Q_{2} \cap\left\{0<-u_{\epsilon}<1 / 2\right\}\right|>\left|Q_{2}\right| / 4$. In such a case, (H3) implies there exists a constant $c$ such that the union of the two cubes contributes at least $c \epsilon^{N \alpha-1} \geqslant c \epsilon^{(N-1) \alpha}$ to the energy.

Case 2: $\left|\mathcal{C} \cap\left\{u_{\epsilon}>1 / 2\right\}\right|,\left|\mathcal{C} \cap\left\{u_{\epsilon}<-1 / 2\right\}\right| \geqslant\left|Q_{1} \cup Q_{2}\right| / 8$. In this case, as in the proof of Lemma 4.2 by applying (3.11) (on two adjacent cubes $\mathcal{C}$ ) and the isoperimetric inequality (Proposition 3.2), we deduce the existence of a constant $c>0$ such that

$$
G\left(u_{\epsilon}, \mathcal{C}\right)-\inf _{H^{1}(\mathcal{C})} G(\cdot, \mathcal{C}) \geqslant c\left(\frac{1}{8} \epsilon^{N \alpha}\right)^{(N-1) / N} .
$$

Hence each such $\mathcal{C}$ contributes at least $c \epsilon^{\alpha(N-1)}$ to the energy. Since each cube has $2 N$ nearest neighbors, we get $\int_{R_{\epsilon}}\left|\nabla\left[H u_{\epsilon}\right]\right| \leqslant C F_{\epsilon}\left(u_{\epsilon}, R_{\epsilon}\right)$. Therefore $H u_{\epsilon}$ is bounded in $B V$ and so it has a subsequence converging strongly in $L^{1}$ to a function $u \in B V$. As a consequence of the lower semicontinuity of the $B V$-norm with respect to $L^{1}$-convergence we obtain $\int_{\mathcal{K}}\left|\nabla\left[H u_{\epsilon}\right]\right| \leqslant$ $C F_{\epsilon}\left(u_{\epsilon}, \Omega\right)$ for any compact set $\mathcal{K} \subset \Omega$. Now $[4.10$ follows by letting $\mathcal{K} \nearrow \Omega$. By [4.12], the corresponding subsequence of the original sequence $u_{\epsilon}$ converges to $u$ as well.

The fact that the $\Gamma$-limit is a measure relies on the following proposition, which is the so-called fundamental estimate [9]. Notice that in our case the proof is quite different from the usual one, due to the fact that $G_{\epsilon}$ is not positive.

Proposition 4.4 Assume (H1)-(H3) and (H5). For any $U, U^{\prime}, V \in \mathcal{A}, U \Subset U^{\prime}$, and for any $u, v \in L_{\text {loc }}^{1}\left(\mathbb{R}^{N}\right)$ there exists a function $\varphi \in C^{\infty}\left(\mathbb{R}^{N},[0,1]\right)$ such that

$$
\varphi=1 \text { on } \bar{U}, \quad \varphi=0 \text { on } \mathbb{R}^{N} \backslash U^{\prime}, \quad|\nabla \varphi| \leqslant C \epsilon^{-1},
$$

and

$$
F_{\epsilon}(\varphi u+(1-\varphi) v, U \cup V) \leqslant F_{\epsilon}\left(u, U^{\prime}\right)+F_{\epsilon}(v, V)+\delta_{\epsilon}\left(u, v, U, U^{\prime}, V\right),
$$

where $\delta_{\epsilon}$ has the property that $\lim _{\epsilon \rightarrow 0} \delta_{\epsilon}\left(u_{\epsilon}, v_{\epsilon}, U, U^{\prime}, V\right)=0$ whenever

$$
\left\{\begin{array}{l}
\left\|u_{\epsilon}-v_{\epsilon}\right\|_{L^{1}(S)} \rightarrow 0, \quad S:=\left(U^{\prime} \backslash \bar{U}\right) \cap V, \\
\sup _{\epsilon}\left\{F_{\epsilon}\left(u_{\epsilon}, U^{\prime}\right)+F_{\epsilon}\left(v_{\epsilon}, V\right)+\left\|u_{\epsilon}\right\|_{\infty}+\left\|v_{\epsilon}\right\|_{\infty}\right\}<\infty .
\end{array}\right.
$$


REMARK 4.5 Assumption (4.15) is stronger than the one made in [9], since we also require $u_{\epsilon}$ and $v_{\epsilon}$ to be bounded in $L^{\infty}\left(\mathbb{R}^{N}\right)$. However, from hypothesis (H3) it follows that we can assume that a $\Gamma$-realizing sequence is bounded in $L^{\infty}$, hence the $\Gamma$-limit does not change if we redefine $F_{\epsilon} \equiv+\infty$ outside a suitable ball of $L^{\infty}\left(\mathbb{R}^{N}\right)$.

Let us define a sequence of strips as follows. Set $U_{0}:=U$ and define by recurrence for each $i \in \mathbb{N}$ :

$$
\begin{aligned}
Z_{i} & :=\left\{z \in \mathbb{Z}^{N}: \epsilon^{\alpha}(Q+z) \subset U^{\prime}, \operatorname{dist}\left(\epsilon^{\alpha}(Q+z), U_{i}\right) \leqslant \epsilon^{\alpha} / 2\right\}, \\
U_{i+1} & :=\bigcup_{z \in Z_{i}} \epsilon^{\alpha}(Q+z), \quad S_{i}:=\left(U_{i+1} \backslash \bar{U}_{i}\right) \cap V .
\end{aligned}
$$

The proof is split in three parts. We start with the following result whose proof is more general than needed, so that it can easily be modified for the case $\alpha \geqslant 1$.

Lemma 4.6 Let $U, U^{\prime}, V, u_{\epsilon}$ and $v_{\epsilon}$ be as in Proposition 4.4. Assume there exist some $S_{i_{0}}$ defined by $4.16, \tilde{S} \subset S_{i_{0}}\left(S_{i_{0}}, \tilde{S} \neq \emptyset\right)$ and $\varphi \in C^{\infty}\left(\mathbb{R}^{N},[0,1]\right)$ such that

$$
\begin{gathered}
F_{\epsilon}\left(u_{\epsilon}, S_{i_{0}}\right)+F_{\epsilon}\left(v_{\epsilon}, S_{i_{0}}\right) \rightarrow 0, \\
\int_{S_{i_{0}}} \frac{\left|u_{\epsilon}-v_{\epsilon}\right|}{\epsilon^{\alpha}} \mathrm{d} x+\int_{\widetilde{S}} \frac{\left|u_{\epsilon}-v_{\epsilon}\right|}{\epsilon} \mathrm{d} x \rightarrow 0, \\
\int_{S_{i_{0}} \backslash \tilde{S}} \frac{W\left(u_{\epsilon}\right)+W\left(v_{\epsilon}\right)}{\epsilon} \mathrm{d} x \rightarrow 0, \\
\int_{S_{i_{0}}} \epsilon\left|\nabla u_{\epsilon}-\nabla v_{\epsilon}\right|^{2} \mathrm{~d} x \rightarrow 0, \quad \int_{S_{i_{0}}} \epsilon\left\{\left|\nabla u_{\epsilon}\right|^{2}+\left|\nabla v_{\epsilon}\right|^{2}\right\} \mathrm{d} x \leqslant C, \\
\operatorname{supp}(\nabla \varphi) \subset \tilde{S}, \quad \varphi=1 \text { on } U_{i_{0}}, \quad \varphi=0 \text { on } \mathbb{R}^{N} \backslash U_{i_{0}+1}, \quad|\nabla \varphi| \leqslant C \epsilon^{-1},
\end{gathered}
$$

where $C$ is independent of $\epsilon$. Then $\lim _{\epsilon \rightarrow 0} F_{\epsilon}\left(\varphi u_{\epsilon}+(1-\varphi) v_{\epsilon}, S_{i_{0}}\right)=0$.

Proof. In order to simplify notation, we shall write $u, v$ instead of $u_{\epsilon}, v_{\epsilon}$ and set $z:=\varphi u+(1-\varphi) v$. We have

$$
\begin{aligned}
F_{\epsilon}\left(z, S_{i_{0}}\right) & =F_{\epsilon}\left(u, S_{i_{0}}\right)+\left\{G_{\epsilon}\left(z, S_{i_{0}}\right)-G_{\epsilon}\left(u, S_{i_{0}}\right)\right\} \\
& =F_{\epsilon}\left(u, S_{i_{0}}\right)+\int_{S_{i_{0}}}\left\{\epsilon\left(|\nabla z|^{2}-|\nabla u|^{2}\right)+\frac{W(z)-W(u)}{\epsilon}+g\left(\frac{x}{\epsilon^{\alpha}}\right) \frac{z-u}{\epsilon^{\alpha}}\right\} \mathrm{d} x \\
& =F_{\epsilon}\left(u, S_{i_{0}}\right)+I_{1}+I_{2}+I_{3} .
\end{aligned}
$$

By 4.17$), F_{\epsilon}\left(u, S_{i_{0}}\right) \rightarrow 0$ while 4.18$)$ implies $I_{3} \rightarrow 0$ (as $\epsilon \rightarrow 0$ ).

For $I_{2}$ we use the fact that $W \in \operatorname{Lip}_{\text {loc }}$, i.e. (H2), together with the inequality $\left\|u_{\epsilon}\right\|_{\infty}+\left\|v_{\epsilon}\right\|_{\infty}$ $\leqslant C$ and the definition of $z$ to get the estimate

$$
\int_{S_{i_{0}}} \frac{W(z)-W(u)}{\epsilon} \mathrm{d} x \leqslant C \int_{\widetilde{S}} \frac{|u-v|}{\epsilon} \mathrm{d} x+\int_{S_{i_{0}} \backslash \tilde{S}} \frac{W(u)+W(v)}{\epsilon} \mathrm{d} x .
$$

Assumptions 4.18) and 4.19) imply that this vanishes as $\epsilon \rightarrow 0$. 
In order to estimate $I_{1}$, note that $\nabla z-\nabla u=\nabla \varphi(u-v)+(1-\varphi)[\nabla(v-u)]$ and $\nabla z+\nabla u=$ $\nabla \varphi(u-v)+\nabla u+\nabla v-\varphi[\nabla(v-u)]$, so we estimate

$$
\begin{aligned}
& \left|\int_{S_{i_{0}}} \epsilon\left(|\nabla z|^{2}-|\nabla u|^{2}\right)\right| \leqslant C\left[\left\|\epsilon^{-1 / 2}|u-v|\right\|_{L^{2}(\widetilde{S})}^{2}+3\left\|\epsilon^{-1 / 2}|u-v|\right\|_{L^{2}(\widetilde{S})}\right. \\
& \left.\cdot\left\|\epsilon^{1 / 2}(|\nabla u|+|\nabla v|)\right\|_{L^{2}\left(S_{i_{0}}\right)}+\left\|\epsilon^{1 / 2}|\nabla u-\nabla v|\right\|_{L^{2}\left(S_{i_{0}}\right)}\left\|\epsilon^{1 / 2}(|\nabla u|+|\nabla v|)\right\|_{L^{2}\left(S_{i_{0}}\right)}\right] .
\end{aligned}
$$

The bound $\left\|u_{\epsilon}\right\|_{\infty}+\left\|v_{\epsilon}\right\|_{\infty} \leqslant C$ allows us to estimate the $L^{2}$-norm by the $L^{1}$-norm, therefore the first term in (4.22) vanishes as $\epsilon \rightarrow 0$ by (4.18), the second by (4.18) and (4.20), and the third by 4.20 .

LEMmA 4.7 Under the assumptions of Proposition 4.4 we can find sets $S_{i_{0}}, \widetilde{S}$ and a function $\varphi$ which fulfill the assumptions of Lemma 4.6

Proof. Since $U \Subset U^{\prime}$, we can assume $U, U^{\prime} \in \mathcal{R}_{\epsilon}$. Consider then the family of $S_{i}$ defined by 4.16). Denote by $k_{\epsilon}$ the largest integer for which $S_{i} \neq \emptyset$ and note that $k_{\epsilon}=O\left(\epsilon^{-\alpha}\right)$.

As the functional is increasing on sets in $\mathcal{R}_{\epsilon}$, the bound on the energy (4.15) allows us to assume that $F_{\epsilon}\left(u_{\epsilon}, S\right)+F_{\epsilon}\left(v_{\epsilon}, S\right) \leqslant C$. Since the functional is additive on disjoint sets in $\mathcal{R}_{\epsilon}$ (see Proposition 3.11, and $\bigcup_{i=0}^{k_{\epsilon}} S_{i} \subset S$, we get

$$
\sum_{i=0}^{k_{\epsilon}}\left\{F_{\epsilon}\left(u_{\epsilon}, S_{i}\right)+F_{\epsilon}\left(v_{\epsilon}, S_{i}\right)\right\} \leqslant F_{\epsilon}\left(u_{\epsilon}, S\right)+F_{\epsilon}\left(v_{\epsilon}, S\right) \leqslant C .
$$

As all terms in the sum are nonnegative, we find that for $2 / 3$ of the indices $i$,

$$
F_{\epsilon}\left(u_{\epsilon}, S_{i}\right)+F_{\epsilon}\left(v_{\epsilon}, S_{i}\right) \leqslant \frac{3 C}{2 k_{\epsilon}}=C^{\prime} \epsilon^{\alpha} .
$$

Such strips satisfy 4.17). The argument used above will be referred to as averaging argument. This averaging argument shows in addition that for $2 / 3$ of the indices $i$,

$$
\int_{S_{i}}\left|u_{\epsilon}-v_{\epsilon}\right| \leqslant C \epsilon^{\alpha} \int_{S}\left|u_{\epsilon}-v_{\epsilon}\right| \text {. }
$$

Hence we can find at least one strip $S_{i_{0}}$ which fulfills both (4.23) and 4.24). There exists a constant $C_{1}$ such that this strip is the disjoint union of at least $C_{1} \epsilon^{\alpha-1}$ strips of the form 4.25 below. So another averaging argument yields a strip $\widetilde{S} \subseteq S_{i_{0}}$ of the form

$$
\widetilde{S}=\left\{x \in U^{\prime}:(j-1) \epsilon \leqslant \operatorname{dist}\left(x, U_{i_{0}}\right) \leqslant j \epsilon\right\} \cap V \quad \text { for some } j \in \mathbb{N},
$$

in which we have

$$
\int_{\widetilde{S}}\left|u_{\epsilon}-v_{\epsilon}\right| \leqslant C_{1} \epsilon^{1-\alpha}\left(C \epsilon^{\alpha} \int_{S}\left|u_{\epsilon}-v_{\epsilon}\right|\right)=C^{\prime} \epsilon \int_{S}\left|u_{\epsilon}-v_{\epsilon}\right| .
$$

As $\left\|u_{\epsilon}-v_{\epsilon}\right\|_{L^{1}(S)} \rightarrow 0$, estimates 4.24 and 4.26 imply (4.18).

Furthermore (4.3), (4.23) and $\left|S_{i_{0}}\right| \leqslant C \epsilon^{\alpha}$ imply (4.19). Moreover using the fact that the renormalization is negative, (4.2) together with (4.23) gives

$$
\int_{S_{i_{0}}} \epsilon\left\{\left|\nabla u_{\epsilon}\right|^{2}+\left|\nabla v_{\epsilon}\right|^{2}\right\} \rightarrow 0,
$$


which implies 4.20 . Finally, from the definition of $\tilde{S}$ given in 4.26 , it is also possible to construct a function $\varphi$ satisfying (4.21).

Proof of Proposition 4.4 Let $i_{0}, S_{i_{0}}$ and $\varphi$ be as in Lemmas 4.6 and 4.7 Since the functionals $F_{\epsilon}$ are additive, setting $z_{\epsilon}:=\varphi u+(1-\varphi) v$ we have

$$
\begin{aligned}
F_{\epsilon}\left(z_{\epsilon}, U \cup V\right)= & F_{\epsilon}\left(z_{\epsilon},(U \cup V) \cap \bar{U}_{i_{0}}\right)+F_{\epsilon}\left(z_{\epsilon},(U \cup V) \cap\left(\mathbb{R}^{N} \backslash U_{i_{0}+1}\right)\right) \\
& +F_{\epsilon}\left(z_{\epsilon},(U \cup V) \cap\left(U_{i_{0}+1} \backslash \bar{U}_{i_{0}}\right)\right) \\
= & F_{\epsilon}\left(u,(U \cup V) \cap \bar{U}_{i_{0}}\right)+F_{\epsilon}\left(v,(U \cup V) \cap\left(\mathbb{R}^{N} \backslash U_{i_{0}+1}\right)\right) \\
& +F_{\epsilon}\left(z_{\epsilon},(U \cup V) \cap\left(U_{i_{0}+1} \backslash \bar{U}_{i_{0}}\right)\right) \\
\leqslant & F_{\epsilon}\left(u, U^{\prime}\right)+F_{\epsilon}(v, V)+F_{\epsilon}\left(z_{\epsilon}, S_{i_{0}}\right) .
\end{aligned}
$$

By Lemma 4.6, $F_{\epsilon}\left(z_{\epsilon}, S_{i_{0}}\right) \rightarrow 0$ as $\epsilon \rightarrow 0$, whenever 4.15 holds.

In the following, we provide some estimates from above and from below for the $\Gamma$-limit, which are useful in order to represent the limit as an integral functional.

Proposition 4.8 Assume that (H1) to (H5) hold and that $g$ is as in Proposition 3.7. Then there exists a constant $C_{3}>0$ such that

$$
\Gamma-\liminf F_{\epsilon}\left(\chi_{E}, \Omega\right) \geqslant C_{3} P(E, \Omega) \quad \forall \Omega \in \mathcal{A}, \forall E \subseteq \Omega .
$$

Proof. Let $\epsilon_{n} \rightarrow 0$ and let $u_{n} \rightarrow \chi_{E}$ in $L^{1}(\Omega)$. Without loss of generality, we may assume that $\liminf _{n \rightarrow \infty} F_{\epsilon_{n}}\left(u_{n}, \Omega\right)<\infty$, hence there exists a subsequence $n_{k}$ such that

$$
\lim _{k \rightarrow \infty} F_{\epsilon_{n_{k}}}\left(u_{n_{k}}, \Omega\right)=\liminf _{n \rightarrow \infty} F_{\epsilon_{n}}\left(u_{n}, \Omega\right)<\infty, \quad \text { and }\left\|u_{\epsilon_{n_{k}}}-\chi_{E}\right\|_{L^{1}} \rightarrow 0 .
$$

Now, 4.10 implies that there exists a $C>0$ such that

$$
\int_{\Omega}\left|\nabla \chi_{E}\right| \leqslant C \lim _{k \rightarrow \infty} F_{\epsilon_{n_{k}}}\left(u_{n_{k}}, \Omega\right)
$$

for a further subsequence (still denoted by $n_{k}$ ). However, by construction,

$$
C \lim _{k \rightarrow \infty} F_{\epsilon_{n_{k}}}\left(u_{n_{k}}, \Omega\right)=C \liminf _{n \rightarrow \infty} F_{\epsilon_{n}}\left(u_{n}, \Omega\right),
$$

which proves the claim.

Proposition 4.9 Assume that (H1) to (H5) hold. Then there exists a constant $C_{2}>0$ such that for any $\Omega \in \mathcal{A}$ with Lipschitz boundary and for any $E \subseteq \Omega$, we have

$$
\Gamma-\lim \sup F_{\epsilon}\left(\chi_{E}, \Omega\right) \leqslant C_{2} P(E, \Omega) .
$$

Proof. By approximating $E$ with regular sets $E_{k}$ such that $P\left(E_{k}, \Omega\right)$ converges to $P(E, \Omega)$, we can assume that $\partial E \cap \Omega$ is a smooth hypersurface. To prove (4.28) it is enough to choose $\epsilon_{n} \rightarrow 0$ and construct a sequence of functions $u_{n} \in H^{1}(\Omega)$ such that

$$
u_{n} \rightarrow \chi_{E} \text { in } L^{1}(\Omega) \text { and } \limsup _{n \rightarrow \infty} F_{\epsilon_{n}}\left(u_{n}, \Omega\right) \leqslant C_{2} P(E, \Omega) .
$$


Let $R_{n} \in \mathcal{R}_{\epsilon_{n}}$ be such that $F_{\epsilon_{n}}(v, \Omega)=F_{\epsilon_{n}}\left(v, R_{n}\right)$ for all $v \in H^{1}(\Omega)$. By Proposition 3.11 , this is the maximal $R \in \mathcal{R}_{\epsilon_{n}}$ which is contained in $\Omega$. The renormalization is given by $\left|R_{n}\right| c_{\epsilon}$. Define

$$
\begin{aligned}
A_{n}^{0} & :=\left\{z \in \mathbb{Z}^{N}: \epsilon_{n}^{\alpha}(Q+z) \subset R_{n}, \operatorname{dist}\left(\epsilon_{n}^{\alpha}(Q+z), \partial E\right)<2 \epsilon_{n}^{\alpha}\right\}, \\
A_{n}^{ \pm} & :=\left\{z \in \mathbb{Z}^{N}: \pm d_{E}\left(\epsilon^{\alpha} z\right) \geqslant 0, \operatorname{dist}\left(\epsilon_{n}^{\alpha}(Q+z), \partial E\right) \geqslant 2 \epsilon_{n}^{\alpha}\right\}, \\
\Sigma_{n} & :=\bigcup_{z \in A_{n}^{0}} \epsilon^{\alpha}(z+Q), \quad R_{n}^{ \pm}:=\bigcup_{z \in A_{n}^{ \pm}} \epsilon^{\alpha}(z+Q) .
\end{aligned}
$$

Consider the positive, periodic minimizer $u_{\epsilon^{1-\alpha}}^{+}$of $\widetilde{G}_{\epsilon^{1-\alpha}}(\cdot, Q)$ on the unit cube. Assumption (H4) implies that the positive and the negative global minimizers differ by the constant 2 . We extend $u_{\epsilon^{1-\alpha}}^{+}$periodically to $\mathbb{R}^{N}$ and denote the extended function by $u_{\epsilon 1-\alpha}^{+}$as well. Consider an even cutoff function $\Phi \in C^{\infty}(\mathbb{R})$, increasing on $[0, \infty)$ and such that $\Phi(r)=0$ if $|r|<1$, and $\Phi(r)=1$ if $|r|>2$.

We denote by $\gamma$ the unique strictly increasing function, asymptotic at $\pm \infty$ to the two stable zeroes \pm 1 of $W$, and satisfying (1.4) with $\gamma(0)=0$. Let $\delta \geqslant 3$ be a fixed natural number such that, if we let $x_{\epsilon}:=\delta|\log \epsilon|$, then $\gamma\left( \pm x_{\epsilon}\right)= \pm 1+O\left(\epsilon^{2 \delta}\right)$ and $\gamma^{\prime}\left( \pm x_{\epsilon}\right)=O\left(\epsilon^{2 \delta}\right)$.

Following [3], we consider a function $\gamma_{\epsilon} \in \mathcal{C}^{1,1}(\mathbb{R}) \cap \mathcal{C}^{\infty}\left(\mathbb{R} \backslash\left\{ \pm x_{\epsilon}, \pm 2 x_{\epsilon}\right\}\right)$ which coincides with $\gamma$ on $\left[-x_{\epsilon}, x_{\epsilon}\right]$ and assumes the asymptotic values \pm 1 outside the interval $\left(-2 x_{\epsilon}, 2 x_{\epsilon}\right)$. Then the sequence

$$
u_{n}(x):=\gamma_{\epsilon_{n}}\left(-\frac{d_{E}(x)}{\epsilon_{n}}\right)+\Phi\left(\frac{d_{E}(x)}{\epsilon_{n}^{\alpha}}\right)\left(u_{\epsilon_{n}^{1-\alpha}}^{+}\left(\frac{x}{\epsilon_{n}^{\alpha}}\right)-1\right)
$$

satisfies $u_{n}=u_{\epsilon_{n}^{1-\alpha}}^{ \pm}\left(x / \epsilon_{n}^{\alpha}\right)$ on $R_{n}^{ \pm}$, if (H4) holds. Since $\partial E$ is regular, there exists a constant $C=$ $C(N)$ such that

$$
\limsup _{n \rightarrow \infty} \frac{\left|\Sigma_{n}\right|}{\epsilon_{n}^{\alpha}} \leqslant C P(E, \Omega) .
$$

Let $v_{n}^{+}(x):=u_{\epsilon^{1-\alpha}}^{+}\left(x / \epsilon_{n}^{\alpha}\right)$. Then the renormalization is given by $G_{\epsilon_{n}}\left(v_{n}^{+}, R_{n}\right)$.

Recalling [3.13], it follows that there exists a constant $C(W)>0$ such that

$$
\left|G_{\epsilon_{n}}\left(v_{n}, \Sigma_{n}\right)\right| \leqslant C P(E, \Omega) \epsilon_{n}^{1-\alpha}+\omega_{n},
$$

where $\omega_{n}$ is such that $\lim _{n} \omega_{n} \epsilon_{n}^{\alpha-1}=0$. As the periodic minimizer $u_{\epsilon^{1-\alpha}}^{+}$is bounded in $L^{\infty}$, we may assume that $\left\|u_{n}\right\|_{\infty} \leqslant 2$. Then we get

$$
\begin{aligned}
F_{\epsilon_{n}}\left(u_{n}, \Omega\right) & =G_{\epsilon_{n}}\left(u_{n}, R_{n}\right)-G_{\epsilon_{n}}\left(v_{n}, R_{n}\right)=G_{\epsilon_{n}}\left(u_{n}, \Sigma_{n}\right)-G_{\epsilon_{n}}\left(v_{n}, \Sigma_{n}\right) \\
& \leqslant \int_{\Sigma_{n}}\left(\epsilon_{n}\left|\nabla u_{n}\right|^{2}+\frac{W\left(u_{n}\right)}{\epsilon_{n}}\right) \mathrm{d} x+\frac{1}{\epsilon_{n}^{\alpha}} \int_{\Sigma_{n}} g\left(\frac{x}{\epsilon_{n}^{\alpha}}\right) u_{n} \mathrm{~d} x+C \epsilon_{n}^{1-\alpha} \\
& \leqslant \int_{\Sigma_{n}}\left(\epsilon_{n}\left|\nabla u_{n}\right|^{2}+\frac{W\left(u_{n}\right)}{\epsilon_{n}}\right) \mathrm{d} x+C\|g\|_{\infty} P(E, \Omega),
\end{aligned}
$$

where $C$ is a constant depending only on $N$. Therefore, recalling [15, 16] we get

$$
\limsup _{n \rightarrow \infty} F_{\epsilon_{n}}\left(u_{n}\right) \leqslant\left(c_{W}+C\|g\|_{\infty}\right) P(E, \Omega) .
$$


REMARK 4.10 Notice that if we drop (H4), we can still show that Proposition 4.9 holds whenever $\alpha<2 / 3$. Indeed, thanks to (H2), (H3) and Proposition 3.7 we get

$$
\left|\widetilde{G}_{\epsilon^{1-\alpha}}\left(u_{\epsilon^{1-\alpha}}^{+}, Q\right)-\widetilde{G}_{\epsilon^{1-\alpha}}\left(u_{\epsilon^{1-\alpha}}^{-}, Q\right)\right| \leqslant C \epsilon^{2(1-\alpha)}
$$

for some $C>0$, which implies that there exists a constant $c_{\epsilon} \geqslant 0$ with lim sup $\epsilon^{\alpha}\left|c_{\epsilon}\right|<\infty$ such that

$$
\min _{H^{1}(R)} G_{\epsilon}(\cdot, R)=\frac{|R|}{\epsilon^{\alpha}} \widetilde{G}_{\epsilon^{1-\alpha}}\left(u_{\epsilon^{1-\alpha}}^{ \pm}, Q\right)=|R|\left(c_{\epsilon}+C \epsilon^{2-3 \alpha}\right)=|R| c_{\epsilon}+o(1) .
$$

Hence we can conclude as above. On the other hand, if $\alpha>2 / 3$ we cannot in general drop (H4) in order to avoid a $\Gamma$-limit which is always in $\{0,+\infty\}$. Indeed, if $W \in C^{3}(\mathbb{R})$, the asymptotic expansion for $u^{ \pm}$shows that

$$
u^{-}(x)-u^{+}(x)=\epsilon^{2(1-\alpha)} \frac{W^{\prime \prime \prime}(1)}{\left(W^{\prime \prime}(1)\right)^{3}} g^{2}(x)+o\left(\epsilon^{2(1-\alpha)}\right),
$$

hence estimate 4.30 is sharp for a general smooth potential.

Once we have both the fundamental estimate and the estimates from above and below, we can reason as in [2, Theorem 3.3] to get the following result.

Proposition 4.11 Assume (H1) to (H5). Then there exists a local functional $F_{0}: L_{\text {loc }}^{1}\left(\mathbb{R}^{N}\right) \times \mathcal{A}$ $\rightarrow[0, \infty]$ and a subsequence of functionals $F_{n_{j}}(\cdot, \Omega)$ which $\Gamma$-converge to $F_{0}(\cdot, \Omega)$ for any $\Omega \in \mathcal{A}$ with Lipschitz boundary. Moreover, for any $u \in B V_{\text {loc }}\left(\mathbb{R}^{N} ;\{-1,1\}\right), F_{0}(u, \cdot)$ is the restriction to $\mathcal{A}$ of a regular Borel measure.

\section{Representation theorem and properties of the $\Gamma$-limit}

In this section we derive further properties of the $\Gamma$-limit. Throughout this section we shall always assume that (H1)-(H5) hold, and that $\|g\|_{L^{N}} \leqslant c_{0}$ with $c_{0}$ as in Proposition 3.7. Let us first introduce the following notation.

Definition 5.1 Let $u_{\epsilon}^{ \pm}$be the periodic extensions of the minimizers of $G_{\epsilon}(\cdot, Q)$, let $\Phi$ and $\gamma_{\epsilon}$ be as in the proof of Proposition 4.9, and let $Q^{v}$ be a unit cube centered at the origin with two of its faces orthogonal to $v$. We set

$$
\begin{aligned}
& H(v, x):=\left\{y \in \mathbb{R}^{N}:\langle y-x, v\rangle \leqslant 0\right\}, \quad \chi^{v, x}:=\chi_{H(v, x)}, \quad Q_{\rho}^{v, x}:=x+\rho Q^{v}, \\
& \bar{u}_{\epsilon}^{v, x}(y):=\gamma_{\epsilon}\left(\frac{d_{H(v, x)}}{\epsilon}\right)+\Phi\left(d_{H(v, x)}\right)\left(u_{\epsilon}^{+}(y)-1\right), \quad \bar{u}_{\epsilon, \alpha}^{v, x}(y):=\bar{u}_{\epsilon^{1-\alpha}}^{v, x}\left(\frac{y}{\epsilon^{\alpha}}\right) .
\end{aligned}
$$

Observe that $\chi^{v, x}$ is the characteristic function of a half-space orthogonal to $v$ and centered at $x$, and $\bar{u}_{\epsilon}^{v, x}(y)$ is an interpolation between the two absolute minimizers across the hyperplane orthogonal to $v$.

Recalling [4, Theorem 3] (see also [2, Theorem 3.5]), we obtain a representation result for the functional $F_{0}$. 
THEOREM 5.2 There exists a function $\varphi: \mathbb{R}^{N} \times \mathcal{S}^{N-1} \rightarrow(0, \infty)$ such that

$$
F_{0}\left(\chi_{E}, B\right)= \begin{cases}\int_{\partial^{*} E \cap B} \varphi\left(x, v_{E}(x)\right) \mathrm{d} \mathcal{H}^{N-1} & \text { if } \chi_{E} \in B V(\Omega), \\ +\infty & \text { otherwise, }\end{cases}
$$

for any $\Omega \in \mathcal{A}$ with Lipschitz boundary and any Borel set $B \subseteq \Omega$. Moreover the function $\varphi$ satisfies

$$
\begin{gathered}
C_{3} \leqslant \varphi(x, v) \leqslant C_{2}, \\
\varphi(x, v)=\limsup _{\rho \rightarrow 0^{+}} \rho^{1-N} m(\rho, x, v),
\end{gathered}
$$

where $C_{2}, C_{3}>0$ are as in Propositions 4.9 and 4.8 while $m(\rho, x, v)$ is defined by

$$
m(\rho, x, v):=\min \left\{F_{0}\left(u, \overline{Q_{\rho}^{v, x}}\right): u=\chi^{\nu, x} \text { in } \mathbb{R}^{N} \backslash Q_{\rho}^{v, x}\right\} .
$$

Relation (5.1) looks slightly different from the formula in [4], but, because of the choice of closed cubes, 5.1) is implied by the result in [4]. More information on $\varphi$ can be extracted from the representation formula 5 5.1), like $x$-independence, convexity and a more explicit representation. To this end, we need two lemmas which allow us to neglect boundary effects. Let us choose a function $u_{\rho}^{\nu, x}$ which solves the minimizing problem defined by $[5.2$, , namely

$$
F_{0}\left(u_{\rho}^{v, x}, \overline{Q_{\rho}^{v, x}}\right)=m(\rho, x, v) .
$$

LEMma 5.3 Given $x \in \mathbb{R}^{N}$, there exists a countable set $\mathcal{E}_{x} \subset \mathbb{R}$ such that, for any $\rho>0$ with $\rho \notin \mathcal{E}_{x}$, there exists a sequence $\eta_{n} \rightarrow \rho, \eta_{n}<\rho$, such that

$$
F_{0}\left(u_{\rho}^{v, x}, \overline{Q_{\rho}^{\nu, x}}\right)=\lim _{n \rightarrow \infty} F_{0}\left(u_{\eta_{n}}^{v, x}, \operatorname{int}\left(Q_{\rho}^{\nu, x}\right)\right) .
$$

Proof. Fix $(v, x) \in \mathcal{S}^{N-1} \times \mathbb{R}^{N}$ and fix $R>0$. To simplify notation, we set $Q_{\rho}:=Q_{\rho}^{\nu, x}$ and $u_{\rho}:=u_{\rho}^{v, x}$ for all $\rho>0$. Let $g_{R}:(0, R) \rightarrow[0, \infty), \eta \mapsto F_{0}\left(u_{\eta}, Q_{R}\right)$. Then $g_{R}$ is a decreasing function on the interval $(0, R)$, hence it has a countable set of discontinuities, $\mathcal{E}_{R}$. Notice that for $R_{1} \leqslant R_{2}$ the two functions $g_{R_{1}}$ and $g_{R_{2}}$ differ by a constant on $\left(0, R_{1}\right)$. Hence $\mathcal{E}_{R_{1}} \subseteq \mathcal{E}_{R_{2}}$ whenever $R_{1} \leqslant R_{2}$. So $\mathcal{E}_{x}=\bigcup_{R>0} \mathcal{E}_{R}$ is countable, and the claim follows.

Lemma 5.4 Let $u_{\rho}^{v, x}$ be as in 5.3. For all $x \in \mathbb{R}^{N}$ and $\rho>0, \rho \notin \mathcal{E}_{x}$, there exist a sequence $\eta_{j} \rightarrow \rho$, with $\eta_{j}<\rho$, and a sequence of functions $u_{j} \rightarrow u_{\rho}^{\nu, x}$ in $L^{1}\left(Q_{\rho}^{\nu, x}\right)$ such that $u_{j} \in H_{\mathrm{loc}}^{1}\left(\mathbb{R}^{N}\right)$, $u_{j}=\bar{u}_{\epsilon_{j}, \alpha}^{v, x}$ on $\mathbb{R}^{N} \backslash Q_{\left(\rho+\eta_{j}\right) / 2}^{v, x}$, and

$$
F_{0}\left(u_{\rho}^{v, x}, \overline{Q_{\rho}^{v, x}}\right)=\lim _{j \rightarrow \infty} F_{\epsilon_{j}}\left(u_{j}, Q_{\rho}^{v, x}\right) .
$$

Proof. As in the proof of the previous lemma, we simplify the notation by dropping the dependence of sets and functions on $x$ and $v$.

By Lemma5.3 we can find a sequence $\eta_{k} \rightarrow \rho, \eta_{k}<\rho$, such that

$$
F_{0}\left(u_{\rho}, \bar{Q}_{\rho}\right)=\lim _{k \rightarrow \infty} F_{0}\left(u_{\eta_{k}}, Q_{\rho}\right),
$$


where $u_{\eta_{k}}=\chi^{\nu, x}$ on $\mathbb{R}^{N} \backslash Q_{\eta_{k}}$. For any $k$, we consider a $\Gamma$-realizing sequence $w_{k, j} \rightarrow u_{\eta_{k}}$ such that

$$
F_{0}\left(u_{\eta_{k}}, Q_{\rho}\right)=\lim _{j \rightarrow \infty} F_{\epsilon_{j}}\left(w_{k, j}, Q_{\rho}\right)
$$

By Proposition 4.4 applied with $U=Q_{\eta_{k}}, U^{\prime}=Q_{\left(\rho+\eta_{k}\right) / 2}, V=Q_{\rho} \backslash \bar{Q}_{\eta_{k}}$ and $u_{\epsilon_{j}}=w_{k, j}$, $v_{\epsilon_{j}}=\bar{u}_{\epsilon_{j}, \alpha}^{\nu, x}$, there exists a cut-off function $\varphi$ between $U$ and $U^{\prime}$. Letting $u_{k, j}:=\varphi u_{\epsilon_{j}}+(1-\varphi) v_{\epsilon_{j}}$, from the energy estimate (4.14) and Proposition 4.9 we obtain

$$
\begin{aligned}
\lim _{j} F_{\epsilon_{j}}\left(u_{k, j}, Q_{\rho}\right) & \leqslant \lim _{j} F_{\epsilon_{j}}\left(w_{k, j}, Q_{\left(\rho+\eta_{k}\right) / 2}\right)+\lim _{j} F_{\epsilon_{j}}\left(\bar{u}_{\epsilon_{j}, \alpha}^{x, v}, Q_{\rho} \backslash \bar{Q}_{\eta_{k}}\right) \\
& \leqslant \lim _{j} F_{\epsilon_{j}}\left(w_{k, j}, Q_{\rho}\right)+C_{2}\left(\rho^{N-1}-\eta_{k}^{N-1}\right) \\
& =F_{0}\left(u_{\eta_{k}}, Q_{\rho}\right)+C_{2}\left(\rho^{N-1}-\eta_{k}^{N-1}\right) .
\end{aligned}
$$

Then a diagonalization argument proves the claim.

REMARK 5.5 Notice that, in Lemma 5.4, we can choose $\eta_{j} \rightarrow \rho$ independently of $\epsilon_{j} \rightarrow 0$; in particular we can assume that for any $k \in \mathbb{N}$ there exists a $j_{0} \in \mathbb{N}$ such that $\eta_{j}<\rho-k \epsilon_{j}^{\alpha}$ for any $j \geqslant j_{0}$.

In the following proposition, we want to show that the $\Gamma$-limit is homogeneous, i.e. the integrand function $\varphi$ does not depend on $x \in \mathbb{R}^{N}$.

Proposition 5.6 The function $\varphi$ given by Theorem 5.2 does not depend on $x$, moreover its onehomogeneous extension $\widetilde{\varphi}$ as defined in 2.5$]$ is convex.

Proof. Let us fix $v \in \mathcal{S}^{N-1}$ and $x, y \in \mathbb{R}^{N}, x \neq y$. We have to show that

$$
\varphi(x, v)=\varphi(y, v) .
$$

Let $u_{\rho}^{x, v}$ be as in (5.3). For simplicity we write $u_{\rho}^{x}:=u_{\rho}^{x, v}$.

Lemma 5.4 asserts the existence of a sequence $u_{j}$ which equals $\bar{u}_{\epsilon_{j}, \alpha}^{v, x}$ on a tubular neighborhood of the boundary of $Q_{\rho}$ and satisfies (5.4). To simplify notation, we drop the dependence of functions and cubes on the direction $v$, which is fixed throughout this proof.

Let $\tau_{j} \in \mathbb{Z}^{N}$ be defined as

$$
\left(\tau_{j}\right)_{i}:=\left[\frac{y_{i}-x_{i}}{\epsilon_{j}}\right]
$$

and $v_{j}(z):=u_{j}\left(z-\epsilon_{j} \tau_{j}\right)$. Here $[r]$ denotes the largest integer smaller than or equal to $r$. Notice that $\tau_{j} \rightarrow y-x$ and $v_{j}(\cdot) \rightarrow v(\cdot):=u_{\rho}^{x}(\cdot-y+x)$. For any $r>1$, we have

$$
\begin{aligned}
F_{0}\left(v, \overline{Q_{\rho}^{y}}\right) & \leqslant F_{0}\left(v, Q_{r \rho}^{y}\right) \leqslant \liminf _{j} F_{\epsilon_{j}}\left(v_{j}, Q_{r \rho}^{y}\right) \\
& =\liminf _{j}\left(F_{\epsilon_{j}}\left(v_{j}, \epsilon_{j} \tau_{j}+Q_{\rho}^{x}\right)+F_{\epsilon_{j}}\left(v_{j}, Q_{r \rho}^{y} \backslash\left(\epsilon_{j} \tau_{j}+Q_{\rho}^{x}\right)\right)\right) \\
& =\liminf _{j}\left(F_{\epsilon_{j}}\left(u_{j}, Q_{\rho}^{x}\right)+F_{\epsilon_{j}}\left(\bar{u}_{\epsilon_{j}}^{x}\left(\cdot-\epsilon_{j} \tau_{j}\right), Q_{r \rho}^{y} \backslash\left(\epsilon_{j} \tau_{j}+Q_{\rho}^{x}\right)\right)\right) \\
& =\lim _{j} F_{\epsilon_{j}}\left(u_{j}, Q_{\rho}^{x}\right)+\lim _{j} F_{\epsilon_{j}}\left(\bar{u}_{\epsilon_{j}}^{x}\left(\cdot-\epsilon_{j} \tau_{j}\right), Q_{r \rho}^{y} \backslash\left(\epsilon_{j} \tau_{j}+Q_{\rho}^{x}\right)\right) \\
& \leqslant F_{0}\left(u_{\rho}^{x}, \overline{Q_{\rho}^{x}}\right)+C_{2} \rho^{N-1}\left(r^{N-1}-1\right) .
\end{aligned}
$$


Letting $r \rightarrow 1$, we then get

$$
F_{0}\left(v, \overline{Q_{\rho}^{y}}\right) \leqslant F_{0}\left(u_{\rho}^{x}, \overline{Q_{\rho}^{x}}\right) .
$$

The choice of $u_{\rho}^{x}$ then implies $m(\rho, y, v) \leqslant m(\rho, x, v)$, where $m(\rho, x, v)$ is defined in 5.2. By exchanging $x$ and $y$, we obtain the equality for any $\rho \notin \mathcal{E}_{x} \cup \mathcal{E}_{y}$. Then, observing that we can rewrite 5.1 in the form

$$
\varphi(x, v)=\limsup _{\rho \rightarrow 0^{+}, \rho \notin \mathcal{E}_{x} \cup \mathcal{E}_{y}} \rho^{1-N} m(\rho, x, v),
$$

we finally get 5.5 .

Once $x$-independence is established, the fact that the extension of $\varphi$ is a convex function follows by standard semicontinuity results (see for example [1]).

REMARK 5.7 Note that if $\varphi$ is independent of $x$, then by dilating the variable $x$ we see that $m(\rho, v)=\rho^{N-1} m(1, v)=\rho^{N-1} \varphi(v)$. In particular the set $\mathcal{E}_{x}$ of discontinuities is empty for any $x \in \mathbb{R}^{N}$. Moreover, by the convexity of $\varphi$, the minimizers $u_{\eta}$ of $m$ are always characteristic functions of a half-space.

We want to prove that the $\Gamma$-limit is independent of the subsequence. In order to do so, it is convenient to work with blow-up sequences and the functional $\widetilde{G}_{\epsilon}$ as in Definition 3.1. We begin by showing that we can choose a suitable minimizing sequence which coincides, far from the interface, with the absolute minimizers on the cube.

First let us introduce some notation. $u_{\epsilon}^{ \pm}$denotes the periodic extension to $\mathbb{R}^{N}$ of the minimizers of $\widetilde{G}_{\epsilon}(\cdot, Q)$. Let $\lambda>0, v \in \mathcal{S}^{N-1}$, and set $\widehat{Q}:=Q^{\nu, 0}$ and

$$
[\lambda \widehat{Q}]:=\bigcup_{\left\{z \in \mathbb{Z}^{N}: Q \subset z+\lambda \widehat{Q}\right\}}(z+\bar{Q}) .
$$

LEMMA 5.8 There exist constants $0<\delta<1 / 3, \epsilon_{0}>0, \lambda_{0}>0$ and $\gamma_{1}>0$ such that for any sequence $u_{\epsilon}$ with boundary values $u_{\epsilon}(x)=\bar{u}_{\epsilon}^{v, 0}(x)$ on $\mathbb{R}^{N} \backslash[\lambda \widehat{Q}]$, which is uniformly bounded in $L^{\infty}$ and satisfies the energy bound

$$
C \lambda^{N-1} \geqslant\left(\widetilde{G}_{\epsilon}\left(u_{\epsilon},[\lambda \widehat{Q}]\right)-\widetilde{G}_{\epsilon}\left(u_{\epsilon}^{ \pm},[\lambda \widehat{Q}]\right)\right),
$$

there exists a sequence $\tilde{u}_{\epsilon}$ with $\tilde{u}_{\epsilon}(x)=u_{\epsilon}(x)$ on $\mathbb{R}^{N} \backslash[\lambda \widehat{Q}]$, and sets $S_{\epsilon}$, which are unions of unit cubes, such that for any $\epsilon<\epsilon_{0}$ and $\lambda>\lambda_{0}$ the following holds:

(a) $\tilde{u}_{\epsilon}=u_{\epsilon}^{+}$or $\tilde{u}_{\epsilon}=u_{\epsilon}^{-}$on $[\lambda \widehat{Q}] \backslash S_{\epsilon}$;

(b) $\widetilde{G}_{\epsilon}\left(\widetilde{u}_{\epsilon},[\lambda \widehat{Q}]\right) \leqslant \widetilde{G}_{\epsilon}\left(u_{\epsilon},[\lambda \widehat{Q}]\right)+C \lambda^{N-1} \epsilon^{\gamma_{1}}$;

(c) $\left|S_{\epsilon} \cap[\lambda \widehat{Q}]\right| \leqslant \epsilon^{-\delta} C \lambda^{N-1}$.

Proof. In the following we will consider $u_{\epsilon}$ as a function on $\mathbb{R}^{N}$, extended by $\bar{u}_{\epsilon}^{v, 0}$ on $\mathbb{R}^{N} \backslash[\lambda \widehat{Q}]$.

Given a constant $0<\gamma<1 / 3$, we set

$$
Z_{\epsilon}^{\gamma}:=\left\{z \in \mathbb{Z}^{N}: \widetilde{G}_{\epsilon}\left(u_{\epsilon}, z+Q\right)-\widetilde{G}_{\epsilon}\left(u_{\epsilon}^{ \pm}, z+Q\right) \geqslant \epsilon^{\gamma}\right\}, \quad S_{\epsilon}^{\gamma}:=\bigcup_{z \in Z_{\epsilon}^{\gamma}}(z+\bar{Q}) .
$$

From the upper bound $\left[5.6\right.$ we have $\left|S_{\gamma}^{\epsilon} \cap[\lambda \widehat{Q}]\right| \leqslant C \lambda^{N-1} \epsilon^{-\gamma}$. 
Fix now a constant $\gamma_{1}<\gamma /[N(N-1)]$ and let

$$
Z_{\epsilon}:=\left\{z \in \mathbb{Z}^{N}: \operatorname{dist}\left(z+Q, S_{\epsilon}^{\gamma}\right) \leqslant 2 \epsilon^{-\gamma_{1}}\right\}, \quad S_{\epsilon}:=\bigcup_{z \in Z_{\epsilon}}(z+\bar{Q}) .
$$

From the boundary conditions we know that $\left|S_{\epsilon} \cap[\lambda \widehat{Q}]\right|>0$. Possibly reducing $\gamma_{1}$, we can also choose $0<\delta<1 / 3$ such that $\gamma+N \gamma_{1}<\delta$. Since we do not have any information on $\mathcal{H}^{N-1}\left(\partial S_{\epsilon}^{\gamma}\right)$, the best available upper bound on $\left|S_{\epsilon}\right|$ is

$$
\left|S_{\epsilon} \cap[\lambda \widehat{Q}]\right| \leqslant C \lambda^{N-1} \epsilon^{-\gamma}\left(\epsilon^{-\gamma_{1}}\right)^{N}=C \lambda^{N-1} \epsilon^{-\left(\gamma+N \gamma_{1}\right)}<C \lambda^{N-1} \epsilon^{-\delta},
$$

and condition (c) is satisfied.

We call a cube positive if $\left|\left\{x \in Q+z: u_{\epsilon}(x)>0\right\}\right| \geqslant 1 / 2$, i.e. if $\left[H u_{\epsilon}\left(\cdot / \epsilon^{\alpha}\right)\right]=1$ on the cube, where $[H u]$ is defined in 4.11 , and negative otherwise. For $x \in \mathbb{R}^{N} \backslash S_{\epsilon}^{\gamma}$, we define $v_{\epsilon}(x)$ by

$$
2 v_{\epsilon}(x):=\left(\left[H u_{\epsilon}\left(\cdot / \epsilon^{\alpha}\right)\right]\left(\epsilon^{\alpha} x\right)+1\right) u_{\epsilon}^{+}(x)+\left(\left[H u_{\epsilon}\left(\cdot / \epsilon^{\alpha}\right)\right]\left(\epsilon^{\alpha} x\right)-1\right) u_{\epsilon}^{-}(x) .
$$

We want to give an estimate of $\left\|u_{\epsilon}-v_{\epsilon}\right\|_{L^{1}\left(\left(S_{\epsilon} \cap[\lambda \widehat{Q}]\right) \backslash S_{\epsilon}^{\gamma}\right)}$.

First we show that there cannot be positive cubes in $\left(S_{\epsilon} \cap[\lambda \widehat{Q}]\right) \backslash S_{\epsilon}^{\gamma}$ which touch negative cubes on one facet. Indeed, assume that we can find two adjacent cubes, say $Q_{1}$ and $Q_{2}$, contained in $S_{\epsilon} \backslash S_{\epsilon}^{\gamma}$, such that $u_{\epsilon}$ is mostly positive in $Q_{1}$ and mostly negative in $Q_{2}$. Note that the energy scales with $\epsilon^{N-1} \alpha$ under the change of variables $y=\epsilon^{-\alpha} x$, so 4.13 implies that there exists a constant $\widehat{C}(W, g)>0$ such that

$$
\widetilde{G}_{\epsilon}\left(u_{\epsilon}, \operatorname{int}\left(\bar{Q}_{1} \cup \bar{Q}_{2}\right)\right) \geqslant \widehat{C} \text {. }
$$

Therefore at least one of the cubes must be in $S_{\epsilon}^{\gamma}$, and $v_{\epsilon}$ is a well-defined $H^{1}$-function on $[\lambda \widehat{Q}] \backslash S_{\epsilon}^{\gamma}$. From 4.5 we get, for $Q_{1}, Q_{2}$ as above,

$$
\left|\left\{u_{\epsilon}<1 / 2\right\} \cap Q_{1}\right| \leqslant C \epsilon^{\gamma N /(N-1)}, \quad\left|\left\{u_{\epsilon}>-1 / 2\right\} \cap Q_{2}\right| \leqslant C \epsilon^{\gamma N /(N-1)} .
$$

By assumptions (H2) and (H3) there is a constant $c$ such that

$$
W(u) \geqslant \begin{cases}c(u-1)^{2} & \text { if } u \geqslant-1 / 2, \\ c(u+1)^{2} & \text { if } u \leqslant 1 / 2 .\end{cases}
$$

Recall that $\widetilde{G}_{\epsilon}\left(u_{\epsilon}^{+}, Q\right) \leqslant 0$ and $\epsilon\left|\nabla u_{\epsilon}\right|^{2}+\epsilon^{-1} W\left(u_{\epsilon}\right) \geqslant 0$. Using 5.8 , we have for $\epsilon$ sufficiently small on a positive cube, which we call for simplicity $Q$,

$$
\begin{aligned}
\epsilon^{\gamma} & >\widetilde{G}_{\epsilon}\left(u_{\epsilon}, Q\right)-\widetilde{G}_{\epsilon}\left(u_{\epsilon}^{+}, Q\right) \\
& \geqslant \int_{\left|u_{\epsilon}-1\right|<3 / 2} \epsilon^{-1}\left[W\left(u_{\epsilon}\right)+\epsilon g\left(u_{\epsilon}-1\right)\right] \mathrm{d} x-\int_{u_{\epsilon}<-1 / 2}\left|g\left(u_{\epsilon}-1\right)\right| \mathrm{d} x \\
& \geqslant-2\|g\|_{L^{\infty} \epsilon^{\gamma N /(N-1)}+} \int_{\left|u_{\epsilon}-1\right|<3 / 2}\left\{\epsilon^{-1} c\left|u_{\epsilon}-1\right|^{2}-\epsilon\|g\|_{\infty}\left|u_{\epsilon}-1\right|\right\} \mathrm{d} x \\
& \geqslant-2\|g\|_{L^{\infty} \epsilon^{\gamma N /(N-1)}}+\int_{2\|g\|_{\infty} \epsilon \leqslant\left|u_{\epsilon}-1\right|<3 / 2}\left\{\epsilon^{-1}(1 / 2)\left|u_{\epsilon}-1\right|^{2}\right\} \mathrm{d} x-2|Q|\|g\|_{L^{\infty}}^{2},
\end{aligned}
$$


hence

$$
\int_{Q \cap\left\{2\|g\|_{\infty} \epsilon \leqslant|u(x)-1|<3 / 2\right\}}|u-1|^{2} \mathrm{~d} x \leqslant C \epsilon^{1+\gamma} .
$$

From (5.8), 5.9), the $L^{\infty}$-bound on $u_{\epsilon}$ and $v_{\epsilon}$ and since $\gamma<1 / 3$ we get

$$
\left\|u_{\epsilon}-v_{\epsilon}\right\|_{L^{1}(Q)}=\left\|u_{\epsilon}-1-\left(v_{\epsilon}-1\right)\right\|_{L^{1}(Q)} \leqslant C\left[\epsilon+\epsilon^{\gamma N /(N-1)}+\epsilon^{(1+\gamma) / 2}\right] \leqslant C \epsilon^{\gamma N /(N-1)},
$$

and the same holds for negative cubes as well. Since $\gamma_{1}<\gamma /[N(N-1)]$ we have $\tau:=\gamma /(N-1)$ $-N \gamma_{1}>0$, so summing over the cubes (see (5.7)) we get

$$
\left\|u_{\epsilon}-v_{\epsilon}\right\|_{L^{1}\left(\left(S_{\epsilon} \cap[\lambda \widehat{Q}]\right) \backslash S_{\epsilon}^{\gamma}\right)} \leqslant C \lambda^{N-1} \epsilon^{\tau} .
$$

In what follows we mimic the proof of the fundamental estimate, with the important difference that the sets are not given, but depend on $\epsilon$.

For $i \in \mathbb{N}, i \leqslant \operatorname{dist}\left([\lambda \widehat{Q}] \backslash S_{\epsilon}, S_{\epsilon}^{\gamma}\right)$, we define the sets $U_{i}$ as follows:

$$
U_{0}:=S_{\epsilon}^{\gamma}, \quad U_{i+1}:=\bigcup_{\left\{z \in \mathbb{Z}^{N}: z+Q \subset S_{\epsilon}, \operatorname{dist}\left(z+Q, U_{i}\right)=0\right\}}(z+\bar{Q}) .
$$

Let also $S_{i}:=U_{i+1} \backslash \bar{U}_{i}$. By the previous $L^{1}$-estimate 5.10 we get

$$
\left|\int_{\left(S_{\epsilon} \cap[\lambda \widehat{Q}]\right) \backslash S_{\epsilon}^{\gamma}} g u_{\epsilon}\right|+\left|\int_{\left(S_{\epsilon} \cap[\lambda \widehat{Q}]\right) \backslash S_{\epsilon}^{\gamma}} g v_{\epsilon}\right| \leqslant C \lambda^{N-1} \epsilon^{\tau} .
$$

(Note that $\int_{A} g \cdot 1=0$ if $A$ is a union of cubes.) This allows us to estimate the nonnegative parts of the functional separately. The idea is to use the upper bound (5.6) and follow the proof of Proposition 4.4

Indeed, 5.11) and (5.6) imply

$$
\int_{\left(S_{\epsilon} \cap[\lambda \widehat{Q}]\right) \backslash S_{\epsilon}^{\gamma}}\left\{\epsilon\left(\left|\nabla u_{\epsilon}\right|^{2}+\left|\nabla v_{\epsilon}\right|^{2}\right)+\epsilon^{-1}\left(W\left(u_{\epsilon}\right)+W\left(v_{\epsilon}\right)\right)\right\} \mathrm{d} x<C \lambda^{N-1} .
$$

Since there are at least $\epsilon^{-\gamma_{1}}$ strips $S_{i}$ contained in $S_{\epsilon} \backslash S_{\epsilon}^{\gamma}$, by an averaging argument we can find $j_{0} \geqslant 1$ such that

$$
\int_{S_{j_{0}} \cap[\lambda \widehat{Q}]}\left\{\epsilon\left(\left|\nabla u_{\epsilon}\right|^{2}+\left|\nabla v_{\epsilon}\right|^{2}\right)+\epsilon^{-1}\left(W\left(u_{\epsilon}\right)+W\left(v_{\epsilon}\right)\right)\right\} \mathrm{d} x<C \lambda^{N-1} \epsilon^{\gamma_{1}} .
$$

Notice that $j_{0} \geqslant 1$, i.e. the chosen strip does not touch the set $S_{0}$. Averaging again, we can also assume

$$
\left\|u_{\epsilon}-v_{\epsilon}\right\|_{L^{1}\left(S_{j_{0}} \cap[\lambda \widehat{Q}]\right)} \leqslant C \lambda^{N-1} \epsilon^{\tau+\gamma_{1}} .
$$

Let us now divide the strip $S_{j_{0}}$ into smaller strips $\Sigma_{j}$ of width $\epsilon$, and let $\varphi_{j}(x)$ be a smooth cut-off function such that $0 \leqslant \varphi_{j} \leqslant 1, \varphi_{j} \equiv 1$ on $V_{j}, \varphi_{j} \equiv 0$ on $[\lambda \widehat{Q}] \backslash V_{j+1}$, where $V_{0}:=U_{i}$, $V_{j+1}:=\left\{x \in U_{i+1}: \operatorname{dist}\left(x, V_{j}\right) \leqslant(j+1) \epsilon\right\}$ and $\Sigma_{j}:=V_{j+1} \backslash \bar{V}_{j}$. Since the boundary of the cubic set $S_{\epsilon}^{\gamma}$ is uniformly Lipschitz, we can also assume $\left|\nabla \varphi_{j}\right| \leqslant C \epsilon^{-1}$ for some $C$ independent of $\epsilon$. We want to choose an index $j$ such that the function

$$
\tilde{u}_{\epsilon}:=\left(1-\varphi_{j}\right) u_{\epsilon}+\varphi_{j} v_{\epsilon}
$$


satisfies condition (b). Notice first that

$$
\begin{aligned}
& \widetilde{G}\left(\tilde{u}_{\epsilon},[\lambda \widehat{Q}]\right)-\widetilde{G}\left(u_{\epsilon},[\lambda \widehat{Q}]\right) \leqslant \widetilde{G}\left(\tilde{u}_{\epsilon}, S_{j_{0}}\right)-\widetilde{G}\left(u_{\epsilon}, S_{j_{0}}\right) \\
& =\int_{S_{j_{0}} \cap[\lambda \widehat{Q}]}\left\{\epsilon\left(\left|\nabla \tilde{u}_{\epsilon}\right|^{2}-\left|\nabla u_{\epsilon}\right|^{2}\right)+\frac{W\left(\tilde{u}_{\epsilon}\right)-W\left(u_{\epsilon}\right)}{\epsilon}+g\left(\tilde{u}_{\epsilon}-u_{\epsilon}\right)\right\} \mathrm{d} x \\
& \leqslant \int_{S_{j_{0}} \cap[\lambda \widehat{Q}]}\left\{\epsilon\left(\left|\nabla \tilde{u}_{\epsilon}\right|^{2}-\left|\nabla u_{\epsilon}\right|^{2}\right)+\frac{W\left(\tilde{u}_{\epsilon}\right)-W\left(u_{\epsilon}\right)}{\epsilon}\right\} \mathrm{d} x+C \lambda^{N-1} \epsilon^{\tau+\gamma_{1}} \text {, }
\end{aligned}
$$

since using 5.13 we have

$$
\int_{S_{j_{0}} \cap[\lambda \widehat{Q}]} g\left(\tilde{u}_{\epsilon}-u_{\epsilon}\right) \mathrm{d} x \leqslant\|g\|_{L^{\infty}}\left\|u_{\epsilon}-v_{\epsilon}\right\|_{L^{1}\left(S_{j_{0}} \cap[\lambda \widehat{Q}]\right)} \leqslant C \lambda^{N-1} \epsilon^{\tau+\gamma_{1}} .
$$

Hence, it remains to prove

$$
\int_{S_{j_{0}} \cap[\lambda \widehat{Q}]}\left\{\epsilon\left(\left|\nabla \tilde{u}_{\epsilon}\right|^{2}-\left|\nabla u_{\epsilon}\right|^{2}\right)+\frac{W\left(\tilde{u}_{\epsilon}\right)-W\left(u_{\epsilon}\right)}{\epsilon}\right\} \mathrm{d} x \leqslant C \lambda^{N-1} \epsilon^{\gamma_{1}} .
$$

Since the number of the smaller strips in $S_{j_{0}}$ is of order $\epsilon^{-1}$, by a further averaging argument and using 5.13, we can find an index $j$ such that

$$
\int_{\Sigma_{j} \cap[\lambda \widehat{Q}]} \frac{\left|u_{\epsilon}-v_{\epsilon}\right|}{\epsilon} \mathrm{d} x \leqslant C \lambda^{N-1} \epsilon^{\tau+\gamma_{1}}
$$

Recalling (5.12) and reasoning as in Proposition 4.4 (estimate 4.22), we obtain

$$
\begin{aligned}
\int_{S_{j_{0}} \cap[\lambda \widehat{Q}]}\left\{\epsilon\left(\left|\nabla \tilde{u}_{\epsilon}\right|^{2}-\left|\nabla u_{\epsilon}\right|^{2}\right)+\right. & \left.\frac{W\left(\tilde{u}_{\epsilon}\right)-W\left(u_{\epsilon}\right)}{\epsilon}\right\} \mathrm{d} x \\
\leqslant & \int_{S_{j_{0} \cap[\lambda \widehat{Q}]}}\left\{\epsilon\left(\left|\nabla v_{\epsilon}\right|^{2}+\left|\nabla u_{\epsilon}\right|^{2}\right)+\frac{W\left(u_{\epsilon}\right)+W\left(v_{\epsilon}\right)}{\epsilon}\right\} \mathrm{d} x \\
& +\int_{\Sigma_{j} \cap[\lambda \widehat{Q}]}\left\{\frac{\left|u_{\epsilon}-v_{\epsilon}\right|^{2}}{\epsilon}+\frac{W\left(\tilde{u}_{\epsilon}\right)-W\left(v_{\epsilon}\right)}{\epsilon}\right\} \mathrm{d} x \\
\leqslant & C \lambda^{N-1} \epsilon^{\gamma_{1}}+C \int_{\Sigma_{j} \cap[\lambda \widehat{Q}]} \frac{\left|u_{\epsilon}-v_{\epsilon}\right|}{\epsilon} \mathrm{d} x \leqslant C \lambda^{N-1} \epsilon^{\gamma_{1}},
\end{aligned}
$$

where we denote by $C$ a general positive constant. By (5.14), this implies

$$
\widetilde{G}\left(\tilde{u}_{\epsilon},[\lambda \widehat{Q}]\right) \leqslant \widetilde{G}\left(u_{\epsilon},[\lambda \widehat{Q}]\right)+C \lambda^{N-1} \epsilon^{\gamma_{1}},
$$

which is condition (b)

It remains to prove that $\tilde{u}_{\epsilon}$ coincides with $u_{\epsilon}$ outside of $[\lambda \widehat{Q}]$. Note that by construction of $v_{\epsilon}$ and the fact that $u_{\epsilon}=\bar{u}_{\epsilon}^{\nu, 0}$ on $\mathbb{R}^{N} \backslash[\lambda \widehat{Q}]$, any cube in $\mathbb{R}^{N} \backslash[\lambda \widehat{Q}]$ such that $u_{\epsilon} \neq v_{\epsilon}$ must be contained in $S_{0} \cup U_{0}$. As $j_{0} \geqslant 1$, we obtain $\tilde{u}_{\epsilon}=u_{\epsilon}$ on $\mathbb{R}^{N} \backslash[\lambda \widehat{Q}]$. 
We show now that the $\Gamma$-limit does not depend on the particular subsequence $\epsilon_{j}$ and on the parameter $\alpha$. In order to do this, we characterize the limit function $\varphi(v)$.

For any Borel set $A \subset \mathbb{R}^{N}$, we define

$$
F_{g}^{A}(E):=c_{W} P(E, A)+\int_{A} g(x) \chi_{E}(x) \mathrm{d} x .
$$

THEOREM 5.9 We have the following representation for the function $\varphi(v)$ :

$$
\begin{aligned}
\varphi(v)=\psi(v):=\liminf _{\lambda \rightarrow \infty} \frac{1}{\lambda^{N-1}} \min \left\{F_{g}^{\left[\lambda Q^{\nu, 0}\right]}(E): E \subseteq \mathbb{R}^{N},\right. & \\
& \left.\chi_{E}=\chi^{\nu, 0} \text { on } \mathbb{R}^{N} \backslash\left[\lambda Q^{\nu, 0}\right]\right\} .
\end{aligned}
$$

In particular, the $\Gamma$-limit does not depend on the subsequence $\epsilon_{j}$ and on the parameter $\alpha \in(0,1)$ 1 Proof. Fix $v \in \mathcal{S}^{N-1}$, set $\widehat{Q}:=Q^{v, 0}$ and let $[\lambda \widehat{Q}]$ be as in Lemma 5.8 We divide the proof into two steps.

Step 1. Let us prove $\varphi \geqslant \psi$. We recall from Lemma 5.4 applied with $x=0$ and $\rho=1$, that

$$
F_{0}\left(\chi^{\nu, 0}, \widehat{Q}\right)=\int_{\partial H(v, 0) \cap \widehat{Q}} \varphi(v) \mathrm{d} \mathcal{H}^{N-1}=\lim _{j \rightarrow \infty} F_{\epsilon_{j}}\left(u_{j}, \widehat{Q}\right),
$$

where $u_{j} \in H_{\mathrm{loc}}^{1}\left(\mathbb{R}^{N}\right)$ are such that $u_{j}=\bar{u}_{\epsilon_{j}, \alpha}^{\nu, 0}$ on $\mathbb{R}^{N} \backslash Q_{\left(1+\eta_{j}\right) / 2}^{\nu, 0}$, for some $\epsilon_{j} \rightarrow 0$ and $\eta_{j} \rightarrow 1$, $\eta_{j}<1$. Notice that we can assume $\eta_{j}<1-4 \epsilon_{j}^{\alpha}$ (see Remark 5.5, which implies that $Q_{\left(1+\eta_{j}\right) / 2}^{v, 0}$ $\left\{x \in \widehat{Q}: \operatorname{dist}\left(x, \mathbb{R}^{N} \backslash[\lambda \widehat{Q}]\right) \geqslant 1\right\} \subseteq[\lambda \widehat{Q}]$.

Let now $\lambda_{j}$ be the greatest integer less than or equal to $\epsilon_{j}^{-\alpha}$, and set $v_{\epsilon_{j}^{1-\alpha}}(x):=u_{j}\left(x / \lambda_{j}\right)$. Since $F_{\epsilon_{j}}\left(u_{j}, \widehat{Q}\right) \leqslant C$ for some $C>0$, it follows that

$$
C \geqslant F_{\epsilon_{j}}\left(u_{j}, \widehat{Q}\right) \geqslant \epsilon_{j}^{(N-1) \alpha}\left(\widetilde{G}_{\epsilon_{j}^{1-\alpha}}\left(v_{\epsilon_{j}^{1-\alpha}},\left[\lambda_{j} \widehat{Q}\right]\right)-\widetilde{G}_{\epsilon_{j}^{1-\alpha}}\left(u_{\epsilon_{j}^{1-\alpha}}^{ \pm},\left[\lambda_{j} \widehat{Q}\right]\right)\right) .
$$

Since $\lambda_{j} \leqslant \epsilon_{j}^{-\alpha} \leqslant \lambda_{j}+1$, from 5.18 it follows that

$$
\widetilde{G}_{\epsilon_{j}^{1-\alpha}}\left(v_{\epsilon_{j}^{1-\alpha}},\left[\lambda_{j} \widehat{Q}\right]\right)-\widetilde{G}_{\epsilon_{j}^{1-\alpha}}\left(u_{\epsilon_{j}^{1-\alpha}}^{ \pm},\left[\lambda_{j} \widehat{Q}\right]\right) \leqslant C \lambda_{j}^{N-1},
$$

possibly for a larger constant $C$.

Set $\widetilde{\epsilon}_{j}:=\epsilon_{j}^{1-\alpha}$. Then the conditions of Lemma 5.8 are satisfied, and we may assume that $v_{\widetilde{\epsilon}_{j}}=u u_{\widetilde{\epsilon}_{j}}^{ \pm}$outside $S_{\widetilde{\epsilon}_{j}}$, for some set $S_{\widetilde{\epsilon}_{j}}$ such that $\left|S_{\widetilde{\epsilon}_{j}} \cap\left[\lambda_{j} \widehat{Q}\right]\right| \leqslant \widetilde{\epsilon}_{j}^{-\delta} C \lambda^{N-1}$ for some $0<\delta<1 / 3$. Fix $\rho>0$ such that $\delta<\rho<1 / 3$. As the renormalization is nonnegative, from the co-area formula we obtain

$$
\begin{aligned}
C \lambda_{j}^{N-1} & \geqslant \widetilde{G}_{\widetilde{\epsilon}_{j}}\left(v_{\widetilde{\epsilon}_{j}}, S_{\widetilde{\epsilon}_{j}} \cap\left[\lambda_{j} \widehat{Q}\right]\right)-\widetilde{G}_{\widetilde{\epsilon}_{j}}\left(u_{\widetilde{\epsilon}_{j}}^{ \pm}, S_{\widetilde{\epsilon}_{j}} \cap\left[\lambda_{j} \widehat{Q}\right]\right) \\
& \geqslant \int_{-1+C \widetilde{\epsilon}_{j}^{\rho}}^{1-C \widetilde{\epsilon}_{j}^{\rho}} \sqrt{W(s)} P\left(\left\{v_{\widetilde{\epsilon}_{j}}>s\right\}, S_{\widetilde{\epsilon}_{j}} \cap\left[\lambda_{j} \widehat{Q}\right]\right) \mathrm{d} s+\int_{S_{\widetilde{\epsilon}_{j}} \cap\left[\lambda_{j} \widehat{Q}\right]} g v_{\widetilde{\epsilon}_{j}} \mathrm{~d} x \\
& \geqslant \int_{-1+C \widetilde{\epsilon}_{j}^{\rho}}^{1-C \widetilde{\epsilon}_{j}^{\rho}} \sqrt{W(s)} P\left(\left\{v_{\widetilde{\epsilon}_{j}}>s\right\}, S_{\widetilde{\epsilon}_{j}} \cap\left[\lambda_{j} \widehat{Q}\right]\right) \mathrm{d} s-2\|g\|_{\infty} \widetilde{\epsilon}_{j}^{-\delta} \lambda_{j}^{N-1} .
\end{aligned}
$$

\footnotetext{
${ }^{1}$ In fact, one can show that the lim inf in (5.17) may be replaced by lim.
} 
Again from Lemma 5.8 we know that $\partial^{*}\left\{v_{\widetilde{\epsilon}_{j}}>s\right\} \subseteq \operatorname{int}\left(S_{\widetilde{\epsilon}_{j}}\right)$ for any $s \in\left[-1+C \widetilde{\epsilon}_{j}^{\rho}, 1-C \widetilde{\epsilon}_{j}^{\rho}\right]$, hence $P\left(\left\{v_{\widetilde{\epsilon}_{j}}>s\right\},{\widetilde{\tilde{\epsilon}_{j}}}_{\widetilde{F}_{j}}\left[\lambda_{j} \widehat{Q}\right]\right)=P\left(\left\{v_{\widetilde{\epsilon}_{j}}>s\right\}\right.$, [ $\left.\left.\lambda_{j} \widehat{Q}\right]\right)$. Let now

$$
t_{j}^{*}:=\arg \min _{-1+C \widetilde{\epsilon}_{j}^{\rho} \leqslant s \leqslant 1-C \widetilde{\epsilon}_{j}^{\rho}} P\left(\left\{v_{\widetilde{\epsilon}_{j}}>s\right\},\left[\lambda_{j} \widehat{Q}\right]\right)
$$

and let

$$
E_{j}^{*}:=\left(\left\{\widetilde{\epsilon}_{\widetilde{j}_{j}}>t_{j}^{*}\right\} \cap\left[\lambda_{j} \widehat{Q}\right]\right)
$$

Then

$$
\int_{-1+C \widetilde{\epsilon}_{j}^{\rho}}^{1-C \widetilde{\epsilon}_{j}^{\rho}} \sqrt{W(s)} P\left(\left\{v_{\widetilde{\epsilon}_{j}}>s\right\},\left[\lambda_{j} \widehat{Q}\right]\right) \mathrm{d} s \geqslant\left(c_{W}-C \widetilde{\epsilon}_{j}^{\rho}\right) P\left(E_{j}^{*},\left[\lambda_{j} \widehat{Q}\right]\right) .
$$

From 5.19, we also know that $P\left(E_{j}^{*},\left[\lambda_{j} \widehat{Q}\right]\right) \leqslant C \lambda_{j}^{N-1} \epsilon^{-\delta}$, hence

$$
\begin{aligned}
\widetilde{G}_{\widetilde{\epsilon}_{j}}\left(v_{\widetilde{\epsilon}_{j}}, S_{\widetilde{\epsilon}_{j}} \cap\left[\lambda_{j} \widehat{Q}\right]\right)-\widetilde{G}_{\widetilde{\epsilon}_{j}}\left(u_{\widetilde{\epsilon}_{j}}^{ \pm}, S_{\widetilde{\epsilon}_{j}} \cap\left[\lambda_{j} \widehat{Q}\right]\right) \\
\quad \geqslant c_{W} P\left(E_{j}^{*},\left[\lambda_{j} \widehat{Q}\right]\right)+\int_{S_{\widetilde{\epsilon}_{j}}} g v_{\widetilde{\epsilon}_{j}} \mathrm{~d} x-C \widetilde{\epsilon}_{j}^{\rho-\delta} \lambda_{j}^{N-1} .
\end{aligned}
$$

Let us now analyze the term $\int_{S_{\widetilde{\epsilon}_{j}} \cap\left[\lambda_{j} \widehat{Q}\right]} g v_{\widetilde{\epsilon}_{j}} \mathrm{~d} x$. For $s \in\left[-1+C \widetilde{\epsilon}_{j}^{\rho}, 1-C \widetilde{\epsilon}_{j}^{\rho}\right]$ we have $W(s) \geqslant$ $c \rho^{2}$ by assumption (H3). This implies that for any $s \in\left[-1+C \tilde{\epsilon}_{j}^{\rho}, 1-C \widetilde{\epsilon}_{j}^{\rho}\right]$,

$$
\left|\left\{v_{\widetilde{\epsilon}_{j}}>s\right\} \triangle E_{j}^{*}\right| \leqslant C \lambda_{j}^{N-1} \widetilde{\epsilon}_{j}^{1-2 \rho-\delta}
$$

since we have the estimate

$$
\begin{aligned}
\left|\left\{v_{\widetilde{\epsilon}_{j}}>s\right\} \Delta E_{j}^{*}\right| C_{0}^{-1} \widetilde{\epsilon}_{j}^{2 \rho-1} & \leqslant \int_{\left\{v_{\tilde{\epsilon}_{j}}>s\right\} \Delta E_{j}^{*}} \frac{W\left(v_{\widetilde{\epsilon}_{j}}\right)}{\widetilde{\epsilon}_{j}} \mathrm{~d} x \\
& \leqslant \widetilde{G}_{\widetilde{\epsilon}_{j}}\left(v_{\widetilde{\epsilon}_{j}}, S_{\widetilde{\epsilon}_{j}} \cap\left[\lambda_{j} \widehat{Q}\right]\right)-G_{\widetilde{\epsilon}_{j}}\left(u_{\widetilde{\epsilon}_{j}}^{ \pm}, S_{\widetilde{\epsilon}_{j}} \cap\left[\lambda_{j} \widehat{Q}\right]\right)-\int_{\widetilde{S}_{\widetilde{\epsilon}_{j}} \cap\left[\lambda_{j} \widehat{Q}\right]} g v_{\widetilde{\epsilon}_{j}} \mathrm{~d} x \\
& \leqslant C \lambda_{j}^{N-1}+C \widetilde{\epsilon}_{j}^{-\delta} \lambda_{j}^{N-1} \leqslant C \widetilde{\epsilon}_{j}^{-\delta} \lambda_{j}^{N-1} .
\end{aligned}
$$

Notice that Proposition 3.4 allows us to assume $\left\|v_{\widetilde{\epsilon}_{j}}\right\| \leqslant 1+C \widetilde{\epsilon}_{j}$. Since $\delta<\rho<1 / 3$, we always have $1-2 \rho>\delta$. It follows that

$$
\begin{aligned}
\int_{S_{\widetilde{\epsilon}_{j}}} g v_{\widetilde{\epsilon}_{j}} \mathrm{~d} x & \geqslant \int_{-1+C \widetilde{\epsilon}_{j}^{\rho}}^{1-C \widetilde{\epsilon}_{j}^{\rho}} \int_{\left\{v_{\widetilde{\epsilon}_{j}}>s\right\} \cap S_{\widetilde{\epsilon}_{j}}} g(x) \mathrm{d} x \mathrm{~d} s-C \widetilde{\epsilon}_{j}^{\rho}\left|S_{\widetilde{\epsilon}_{j}}\right| \\
& \geqslant 2 \int_{E_{j}^{*} \cap S_{\widetilde{\epsilon}_{j}}} g(x) \mathrm{d} x-2\|g\|_{\infty} C \lambda_{j}^{N-1} \widetilde{\epsilon}_{j}^{1-2 \rho-\delta}-C \widetilde{\epsilon}_{j}^{\rho-\delta} \lambda_{j}^{N-1} .
\end{aligned}
$$

Notice that

$$
\int_{E_{j}^{*} \cap S_{\tilde{\epsilon}_{j}}} g(x) \mathrm{d} x=\int_{E_{j}^{*} \cap\left[\lambda_{j} \widehat{Q}\right]} g(x) \mathrm{d} x,
$$


hence from 5.20) and 5.21, observing that $\lim _{j \rightarrow \infty} \epsilon_{j}^{\alpha} \lambda_{j}=1$, we obtain

$$
F_{\epsilon_{j}}\left(u_{j}, \widehat{Q}\right) \geqslant \frac{\left(\epsilon_{j}^{\alpha} \lambda_{j}\right)^{N-1}}{\lambda_{j}^{N-1}}\left(c_{W} P\left(E_{j}^{*},\left[\lambda_{j} \widehat{Q}\right]\right)+2 \int_{E_{j}^{*} \cap\left[\lambda_{j} \widehat{Q}\right]} g(x) \mathrm{d} x-C \lambda_{j}^{N-1} \epsilon_{j}^{(1-\alpha)(\rho-\delta)}\right) .
$$

We now modify the sets $E_{j}^{*}$ in such a way that $\chi_{E_{j}^{*}}=\chi^{v, 0}$ on $\mathbb{R}^{N} \backslash\left[\lambda_{j} \widehat{Q}\right]$. Let

$$
\partial_{1}\left(\lambda_{j} \widehat{Q}\right):=\left\{x \in\left[\lambda_{j} \widehat{Q}\right]: \operatorname{dist}\left(x, \mathbb{R}^{N} \backslash\left[\lambda_{j} \widehat{Q}\right]\right) \leqslant 1\right\} .
$$

Since $v_{\widetilde{\epsilon}_{j}}=\bar{u}_{\widetilde{\epsilon}_{j}}^{\nu, 0}$ on $\partial_{1}\left(\lambda_{j} \widehat{Q}\right)$, we have

$$
\max _{x \in \partial E_{j}^{*} \cap \partial_{1}\left(\lambda_{j} \widehat{Q}\right)} \operatorname{dist}(x, \partial H(\nu, 0)) \leqslant 2, \quad j \in \mathbb{N} .
$$

Hence, we can find a set $\widehat{E}_{j}^{*}$ which coincides with $E_{j}^{*}$ on $\left[\lambda_{j} \widehat{Q}\right] \backslash \partial_{1}\left(\lambda_{j} \widehat{Q}\right)$ and with $H(\nu, 0)$ on $\mathbb{R}^{N} \backslash\left[\lambda_{j} \widehat{Q}\right]$, such that

$$
\left|E_{j}^{*} \triangle \widehat{E}_{j}^{*}\right|+\left|P\left(E_{j}^{*},\left[\lambda_{j} \widehat{Q}\right]\right)-P\left(\widehat{E}_{j}^{*},\left[\lambda_{j} \widehat{Q}\right]\right)\right| \leqslant C \lambda^{N-2} .
$$

We can finally conclude

$$
\begin{aligned}
& \varphi(\nu)= F_{0}\left(\chi_{E}, \widehat{Q}\right)=\lim _{j} F_{\epsilon_{j}}\left(u_{j}, \widehat{Q}\right) \\
& \qquad \liminf _{\substack{\lambda \rightarrow+\infty \\
\lambda \in \mathbb{N}}} \frac{1}{\lambda^{N-1}} \min \left\{c_{W} P(E,[\lambda \widehat{Q}])+2 \int_{E \cap[\lambda \widehat{Q}]} g(x) \mathrm{d} x:\right. \\
&\left.\qquad E \subseteq \mathbb{R}^{N}, \chi_{E}=\chi^{\nu, 0} \text { on } \mathbb{R}^{N} \backslash[\lambda \widehat{Q}]\right\}=\psi(v) .
\end{aligned}
$$

Step 2. Let us prove $\varphi \leqslant \psi$. Since finite perimeter sets can be approximated by smooth sets in $L^{1}$ and in perimeter (see e.g. [12, Theorem 1.24]), we can choose a sequence $\lambda_{j} \rightarrow \infty$ and sets $E_{j} \subset \mathbb{R}^{N}$ of class $\mathbf{C}^{\infty}$ and $E_{j}=H(\nu, 0)$ outside $\left[\lambda_{j} \widehat{Q}\right]$ such that

$$
\psi(v)=\lim _{j \rightarrow \infty} \frac{1}{\lambda_{j}^{N-1}}\left(c_{W} P\left(E_{j},\left[\lambda_{j} \widehat{Q}\right]\right)+\int_{\left[\lambda_{j} \widehat{Q}\right]} g(x) \chi_{E_{j}} \mathrm{~d} x\right) .
$$

Notice that, without requiring further regularity on $g$, we do not have estimates on the second fundamental form of $\partial E_{j}$.

From [8, Section 11] (which can be adapted to the case $g \in L^{\infty}$ ) it follows that there exist a set $E \subset \mathbb{R}^{N}$ and a constant $k=k(g)>0$ such that

$$
\sup _{x \in \partial E} \operatorname{dist}\left(x, \partial H^{v, 0}\right) \leqslant k, \quad j \in \mathbb{N},
$$

and for any compact set $K \subseteq \mathbb{R}^{N}$,

$$
F_{g}(E, K) \leqslant F_{g}(\tilde{E}, K) \quad \text { if } \tilde{E}=E \text { on } \mathbb{R}^{N} \backslash K .
$$


Moreover, if $v$ has rational coordinates, then $E$ is periodic under translation by any vector $k \in \mathbb{Z}^{N}$ with $k \cdot v=0$. From Proposition 5.6 we know that $\varphi$ is convex, hence continuous in $v$. Therefore we may assume without loss of generality that $v$ has rational coordinates.

In this case, we can use the periodic sets $E$ from [8, Section 11] to construct a minimizing sequence $E_{j}$ for 5.17, which is made up of $C(v) \lambda_{j}^{N-1}$ copies of a fixed surface. Note that the error introduced by the slightly different boundary conditions (in a strip around a plane) is of order $\lambda_{j}^{N-2}$.

As a consequence, we can approximate this minimizing sequence by a sequence $\widehat{E}_{j}$ of sets such that $\partial \widehat{E}_{j}$ is of class $\mathbf{C}^{2}$ and

$$
\left|P\left(E_{j},[\lambda \widehat{Q}]\right)-P\left(\widehat{E}_{j},[\lambda \widehat{Q}]\right)\right|+\left|F_{g}\left(E_{j},[\lambda \widehat{Q}]\right)-F_{g}\left(\widehat{E}_{j},[\lambda \widehat{Q}]\right)\right| \leqslant \delta_{j} \lambda_{j}^{N-1},
$$

and the second fundamental form of $\widehat{E}_{j}$ is bounded by a constant $C\left(\delta_{j}\right)$.

We now reason as in the proof of Proposition 4.9 and we construct a sequence of functions $u_{j}$ defined as in (4.29) with $E$ replaced by $\widehat{E}_{j}, \epsilon_{j}=\lambda_{j}^{-1 / \alpha}$ and $R_{j}=\left[\lambda_{j} \widehat{Q}\right] / \lambda_{j} \subseteq \widehat{Q}$. Notice that $u_{j}$ coincides with $\chi^{\nu, 0}$ outside $\widehat{Q}$ and that from 5.23 it follows that $u_{j} \rightarrow \chi^{\nu, 0}$ in $L^{1}\left(\mathbb{R}^{N}\right)$. We let

$$
Z_{j}:=\left\{z \in \mathbb{Z}^{N}: Q+z \subset\left[\lambda_{j} \widehat{Q}\right], \operatorname{dist}\left(Q+z, \partial \widehat{E}_{j}\right)<2\right\}, \quad \Sigma_{j}:=\bigcup_{z \in Z_{j}}(\bar{Q}+z) .
$$

By 5.23 , we know that $\left|\Sigma_{j}\right| \leqslant 4(k+1) \lambda_{j}^{N-1}$. Notice that, letting

$$
v_{j}:=\gamma_{\epsilon_{j}^{1-\alpha}}\left(d_{\widehat{E}_{j}} / \epsilon_{j}^{1-\alpha}\right)
$$

and recalling [15, 16], there exists a constant $C$, depending only on the norm of the second fundamental form of $\partial \widehat{E}_{j}$, such that

$$
\int_{\left[\lambda_{j} \widehat{Q}\right]}\left(\epsilon_{j}^{1-\alpha}\left|\nabla v_{j}\right|^{2}+\frac{W\left(v_{j}\right)}{\epsilon_{j}^{1-\alpha}}\right) \mathrm{d} x \leqslant\left(1+C \epsilon_{j}^{1-\alpha}\right) c_{W} P\left(\widehat{E}_{j},\left[\lambda_{j} \widehat{Q}\right]\right) .
$$

Following the computations in the proof of Proposition 4.9 we thus obtain

$$
\begin{aligned}
\liminf _{j \rightarrow \infty}\left(G_{\epsilon_{j}}\left(u_{j}, \widehat{Q}\right)-c_{\epsilon_{j}}\right) \leqslant & \liminf _{j \rightarrow \infty} \frac{1}{\lambda_{j}^{N-1}}\left(c_{W} P\left(\widehat{E}_{j},\left[\lambda_{j} \widehat{Q}\right]\right)+\int_{\Sigma_{j}} g(x) u_{j}\left(\lambda_{j} x\right) \mathrm{d} x\right)+\delta_{j} \\
= & \liminf _{j \rightarrow \infty} \frac{1}{\lambda_{j}^{N-1}}\left(c_{W}\left(1+C\left(\delta_{j}\right) \epsilon_{j}^{1-\alpha}\right) P\left(E_{j},\left[\lambda_{j} \widehat{Q}\right]\right)\right. \\
& \left.+\int_{E_{j}} g(x) \mathrm{d} x+\int_{\Sigma_{j}} g(x)\left(u_{j}\left(\lambda_{j} x\right)-\chi_{E_{j}}(x)\right) \mathrm{d} x\right)+\delta_{j} .
\end{aligned}
$$

Define

$$
\widetilde{\Sigma}_{j}:=\left\{x \in\left[\lambda_{j} \widehat{Q}\right]: \operatorname{dist}\left(x, \partial \widehat{E}_{j}\right)<2 \epsilon_{j}^{1-\alpha} \log \left(\epsilon_{j}^{1-\alpha}\right)\right\} .
$$

Notice that $\left|\widetilde{\Sigma}_{j}\right| \leqslant C P\left(\widehat{E}_{j},\left[\lambda_{j} \widehat{Q}\right]\right) \epsilon_{j}^{1-\alpha} \log \left(\epsilon_{j}\right)$ and, similarly, $\left|\Sigma_{j}\right| \leqslant C P\left(\widehat{E}_{j},\left[\lambda_{j} \widehat{Q}\right]\right)$. 
By definition of $u_{j}$ we have

$$
\begin{aligned}
\left|\int_{\Sigma_{j}} g(x)\left(u_{j}\left(\lambda_{j} x\right)-\chi_{\widehat{E}_{j}}(x)\right) \mathrm{d} x\right| \leqslant & \left.\int_{\widetilde{\Sigma}_{j}} \mid g(x) u_{j}\left(\lambda_{j} x\right)-\chi_{\widehat{E}_{j}}(x)\right) \mid \mathrm{d} x \\
& +\int_{\Sigma_{j} \backslash \widetilde{\Sigma}_{j}}|g(x)|\left|u_{j}\left(\lambda_{j} x\right)-\chi_{\widehat{E}_{j}}(x)\right| \mathrm{d} x \\
\leqslant & C\left|\widetilde{\Sigma}_{j}\right|+C\left|\Sigma_{j}\right| \epsilon_{j}^{1-\alpha} \leqslant C \lambda_{j}^{N-1} \epsilon_{j}^{1-\alpha} \log \left(\epsilon_{j}\right) .
\end{aligned}
$$

It follows that

$$
\varphi(v) \leqslant \liminf _{j \rightarrow \infty} F_{\epsilon_{j}}\left(u_{j}, \widehat{Q}\right) \leqslant \liminf _{j \rightarrow \infty} \frac{1}{\lambda_{j}^{N-1}}\left(c_{W} P\left(E_{j},\left[\lambda_{j} \widehat{Q}\right]\right)+\int_{\widehat{E}_{j}} g(x) \mathrm{d} x\right)=\psi(v)
$$

for an appropriate choice of $\delta_{j} \rightarrow 0$.

REMARK 5.10 We point out that, if $N=2$, the results from [8] are not needed, since any minimizer of (5.17) has boundary of class $\mathbf{C}^{1,1}$, with curvature bounded by $\|g\|_{\infty}$.

We conclude this section by showing that the presence of the function $g$ has always the effect of decreasing the energy of the limit functional.

Proposition 5.11 We have

$$
\varphi(v)=\varphi(-v) \leqslant c_{W} \quad \forall v \in \mathcal{S}^{N-1} .
$$

Proof. Let $\delta>0$. Note that $-\chi^{v, 0}=\chi^{-v, 0}$, so the representation formula 5.17) asserts the existence of a $\lambda_{\delta}>0$ such that for $\lambda>\lambda_{\delta}$,

$$
\begin{gathered}
\varphi(\nu) \leqslant c_{W}+\lambda^{1-N} \int_{[\lambda \widehat{Q}]} \chi^{\nu, 0} g(x) \mathrm{d} x+\delta, \\
\varphi(-\nu) \leqslant c_{W}-\lambda^{1-N} \int_{[\lambda \widehat{Q}]} \chi^{\nu, 0} g(x) \mathrm{d} x+\delta .
\end{gathered}
$$

Adding these equations and letting $\delta \rightarrow 0$ we see that the symmetric part $\varphi_{S}$ of $\varphi$ satisfies

$$
\varphi_{S}(v)=\frac{1}{2}(\varphi(v)+\varphi(-v)) \leqslant c_{W} .
$$

The symmetry condition on $g$ yields, in particular, $g(x)=g(-x)$, hence

$$
\int_{[\lambda \widehat{Q}]} g(x) \chi_{E}(x) \mathrm{d} x=\int_{[\lambda \widehat{Q}]} g(-x) \chi_{E}(x) \mathrm{d} x=\int_{[\lambda \widehat{Q}]} g(x) \chi_{E}(-x) \mathrm{d} x .
$$

Notice that $\chi^{\nu, 0}(-x)=-\chi^{\nu, 0}(x)=\chi^{-\nu, 0}(x)$, therefore $\chi_{E}(x)=\chi^{\nu, 0}(x)$ on $\mathbb{R}^{N} \backslash[\lambda \widehat{Q}]$ implies $\chi_{E}(-x)=\chi^{-v, 0}(x)$ on $\mathbb{R}^{N} \backslash[\lambda \widehat{Q}]$. From (5.17) and [5.26) it then follows that $\varphi(v)=\varphi(-v)$, which gives the assertion together with (5.25).

Notice that Theorem 2.3 follows directly from Proposition 4.11. Theorem 5.2 Proposition 5.6. Theorem 5.9 and Proposition 5.11 . 


\section{REFERENCES}

1. Ambrosio, L., \& Braides, A. Functionals defined on partitions in sets of finite perimeter I, II. J. Math. Pures Appl. 69 (1990), 285-333. Zbl 0676.49028 MR 1070481

2. Ansini, N., BRAIDES, A., \& ChiAdò PiAT, V. Gradient theory of phase transitions in composite media. Proc. Roy. Soc. Edinburgh Sect. A 133 (2003), 265-296. Zbl 1031.49021 MR 1969814

3. Bellettini, G., \& PaOlini, M. Quasi-optimal error estimates for the mean curvature flow with a forcing term. Differential Integral Equations 8 (1995), 735-752. Zbl 0820.49019 MR 1306590

4. Bouchitté, G., Fonseca, I., Leoni, G., \& Mascarenhas, L. A global method for relaxation in $W^{1, p}$ and $S B V_{p}$. Arch. Rat. Mech. Anal. 165 (2002), 187-242. Zbl 1028.49009 MR 1941478

5. Braides, A. Approximation of Free-Discontinuity Problems. Lecture Notes in Math. 1694, Springer, (1998). Zbl 0909.49001 MR 1651773

6. Braides, A., \& Defranceschi, A. Homogenization of Multiple Integrals. Oxford Univ. Press, Oxford (1998). Zbl 0911.49010 MR 1684713

7. Braides, A., \& Truskinovsky, L. $\Gamma$-convergence of parametrized functionals. Preprint, Univ. of Rome II (2005).

8. Caffarelli, L., \& De la Llave, R. Plane-like minimizers in periodic media. Comm. Pure Appl. Math. 54 (2001), 1403-1441. Zbl 1036.49040 MR 1852978

9. Dal Maso, G. An Introduction to $\Gamma$-convergence. Birkhäuser, Boston (1993). Zbl 0816.49001 MR 1201152

10. De Giorgi, E. Sulla convergenza di alcune successioni di integrali del tipo dell'area. Rend. Mat. 8 (1975), 277-294. Zbl 0316.35036 MR 0375037

11. Evans, L. C., \& Gariepy, R. L. Measure Theory and Fine Properties of Functions. Stud. Adv. Math., CRC Press, Boca Raton, 1992. Zbl 0804.28001 MR 1158660

12. GiUsti, E. Minimal Surfaces and Functions of Bounded Variation. Birkhäuser, Boston (1984). Zbl 0545.49018 MR 0775682

13. Hutchinson, J. E., \& Tonegawa, Y. Convergence of phase interfaces in the Van der WaalsCahn-Hilliard theory. Calc. Var. Partial Differential Equations 10 (2000), 49-84. Zbl 1070.49026 MR 1803974

14. Massari, U. Esistenza e regolarità delle ipersuperfici di curvatura media assegnata in $\mathbb{R}^{n}$. Arch. Rat. Mech. Anal. 55 (1974), 357-382. Zbl 0305.49047 MR 0355766

15. ModicA, L. The gradient theory of phase transitions and the minimal interface criterion. Arch. Rat. Mech. Anal. 98 (1987), 123-142. Zbl 0616.76004 MR 0866718

16. Modica, L., \& Mortola, S. Un esempio di $\Gamma^{-}$-convergenza. Boll. Un. Mat. Ital. B 14 (1977), 285299. Zbl 0356.49008 MR 0445362

17. Ziemer, W. P. Weakly Differentiable Functions. Grad. Texts in Math. 120, Springer, New York (1989). Zbl 0692.46022 MR 1014685 